\title{
A path algorithm for the Fused Lasso Signal Approximator
}

\author{
Holger Hoefling* \\ Stanford University
}

October 3, 2009

\begin{abstract}
The Lasso is a very well known penalized regression model, which adds an $L_{1}$ penalty with parameter $\lambda_{1}$ on the coefficients to the squared error loss function. The Fused Lasso extends this model by also putting an $L_{1}$ penalty with parameter $\lambda_{2}$ on the difference of neighboring coefficients, assuming there is a natural ordering. In this paper, we develop a fast path algorithm for solving the Fused Lasso Signal Approximator that computes the solutions for all values of $\lambda_{1}$ and $\lambda_{2}$. In the supplement, we also give an algorithm for the general Fused Lasso for the case with predictor matrix $\mathbf{X} \in \mathbb{R}^{n \times p}$ with $\operatorname{rank}(\mathbf{X})=p$.
\end{abstract}

\section{Introduction}

In recent years, many regression procedures have been proposed that use penalties on the regression coefficients in order to achieve sparseness or shrink them towards zero. One of the most widely known procedures of this type is the Lasso (see Tibshirani [1996]), which minimizes the loss function

$$
\frac{1}{2}(\mathbf{y}-\mathbf{X} \boldsymbol{\beta})^{T}(\mathbf{y}-\mathbf{X} \boldsymbol{\beta})+\lambda_{1} \sum_{i=1}^{p}\left|\beta_{i}\right| .
$$

Here, $\mathbf{y} \in \mathbb{R}^{n}$ is the response vector, $\mathbf{X} \in \mathbb{R}^{n \times p}$ is the matrix of predictors and $\boldsymbol{\beta} \in \mathbb{R}^{p}$ the coefficient vector. Several years after the original Lasso paper was published, the LARS algorithm was developed (see Efron et al. [2004]), which after a small adjustment gives the whole solution path of the Lasso for the penalty parameter $\lambda_{1}$ with the computational complexity of an ordinary least squares problem. Subsequently, path algorithms for several other regression methods were developed as well, for example for generalized linear models (see Park and Hastie [2007]) or the SVM (see Hastie et al. [2004]) among others. A more general treatment of conditions under which the solution paths are piecewise linear can be found in Rosset and Zhu [2007].

An example of an extension of the Lasso is the Fused Lasso introduced in Tibshirani et al. [2005]. For the Fused Lasso, it is assumed that there is some natural ordering of the

*Email: hhoeflin@gmail.com 
coefficients (e.g. each coefficient corresponds to a position on a straight line). If coefficients in the true model are closely related to their neighbors, we can exploit this by placing an additional penalty on the differences of neighboring coefficients. Several different choices for these penalties on neighboring coefficients are possible and in the case of the Fused Lasso, an $L_{1}$ penalty is being used. The resulting loss function is then

$$
\frac{1}{2}(\mathbf{y}-\mathbf{X} \boldsymbol{\beta})^{T}(\mathbf{y}-\mathbf{X} \boldsymbol{\beta})+\lambda_{1} \sum_{i=1}^{p}\left|\beta_{i}\right|+\lambda_{2} \sum_{i=1}^{p-1}\left|\beta_{i}-\beta_{i+1}\right| .
$$

The second penalty with parameter $\lambda_{2}$ shrinks neighboring coefficients towards each other. Just as the $L_{1}$ penalty on the absolute values $\left|\beta_{i}\right|$ for the Lasso encourages sparseness, the penalty on $\left|\beta_{i}-\beta_{i+1}\right|$ tends to set neighboring penalties exactly equal to each other. As such, the method is especially suitable for coefficients that are constant for an interval and change in jumps.

In this article, we first want to concentrate on the most widely used case for this method, the Fused Lasso Signal Approximator (FLSA). In the FLSA, we assume that we have $\mathbf{X}=\mathbf{I}$ as the predictor matrix. One example for this would be comparative genomic hybridization (CGH) or chromosomal microarray analysis (CMA) data. CGH is a method that identifies DNA copy number gains and losses on chromosomes by making two color fluorescence in situ hybridization at various points of the chromosomes. In this technique, normal and tumor DNA are labeled with fluorescent dyes (e.g. red and green) and using a microarray analysis, regions of increased or decreased fluorescence of one color compared to the other can be identified, indicating gains or losses of DNA at this place of the chromosome. As usual with this type of data, it is very noisy. Therefore, we seek to exploit that gains or losses typically appear for whole regions in the genome and that these changes usually occur in jumps. We can do this by penalizing differences of neighboring coefficients and therefore decrease the noise in the data and improve estimation. In this case, we use the one-dimensional Fused Lasso Signal Approximator (FLSA), for which the loss function is

$$
L(\mathbf{y}, \boldsymbol{\beta})=\frac{1}{2} \sum_{i=1}^{n}\left(y_{i}-\beta_{i}\right)^{2}+\lambda_{1} \sum_{i=1}^{n}\left|\beta_{i}\right|+\lambda_{2} \sum_{i=1}^{n-1}\left|\beta_{i}-\beta_{i+1}\right| .
$$

Every coefficient $\beta_{i}$ is an estimate of the measurement $y_{i}$ taken at position $\mathrm{i}$ (which we assume to be ordered along the chromosome). Apart from the Lasso penalty $\lambda_{1} \sum_{i=1}^{n}\left|\beta_{i}\right|$, the additional penalty placed on the difference between neighboring coefficients is $\lambda_{2} \sum_{i=1}^{n-1} \mid \beta_{i}-$ $\beta_{i+1} \mid$. An example of CGH measurements in lung cancer can be seen in Figure 1 . The red line are the estimates for penalty parameters $\lambda_{1}=0$ and $\lambda_{2}=2$. We can see that starting around measurement 150 , the $\mathrm{CGH}$ results are on average below 0 , indicating a loss of DNA in this region.

Another example where the Fused Lasso model can be used is in image reconstruction. As a toy example, look at Figure 2. On the left hand side, we can see the true image and a noisy version in the middle. On the right hand side is the denoised version using the Fused Lasso. As the coefficients are not located on a straight line but instead on a 2-D grid, we have to use a different version of the penalty that penalizes all differences of neighboring coefficients in 2 dimensions.

In its more general form, we assume that each coefficient corresponds to a node in a graph 


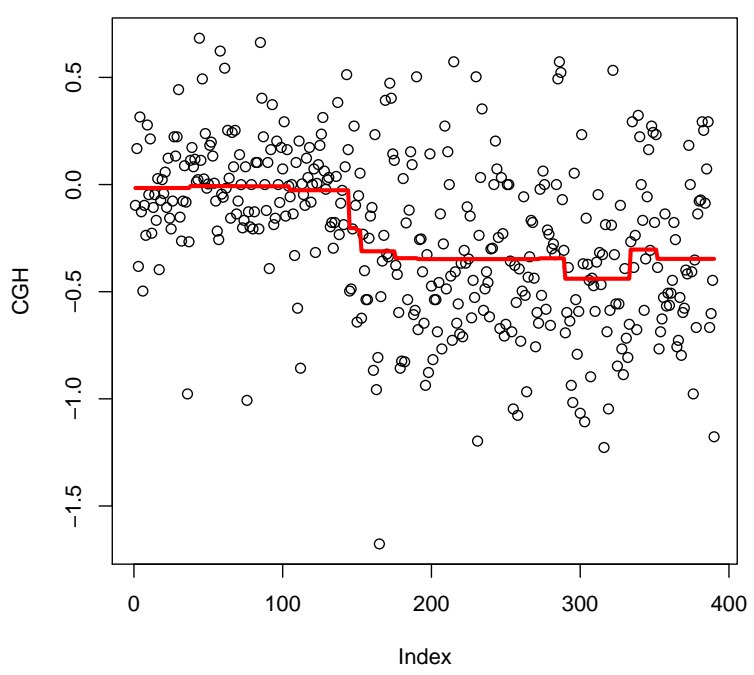

Figure 1: Example using the one-dimensional Fused Lasso Signal Approximator on lung cancer CGH data.
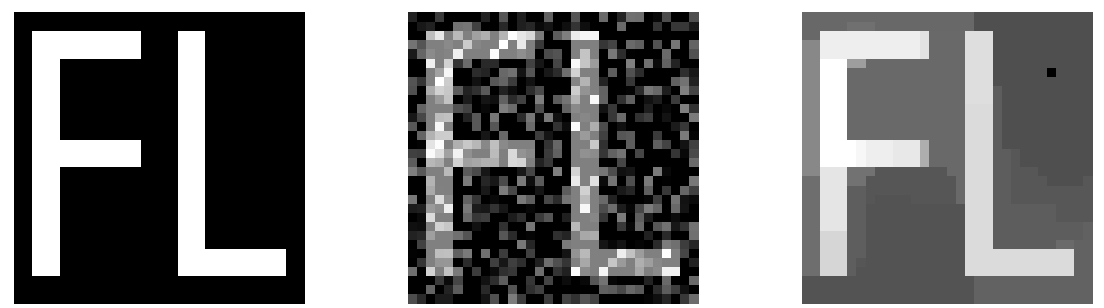

Figure 2: Example of image reconstruction using the Fused Lasso. On the left hand side is the true image. The noisy version is in the middle. The reconstructed version using the Fused Lasso is on the right.

$\mathcal{G}=(V, E)$. Then we penalize every difference of coefficients if the corresponding nodes have an edge between them. Specifically, the loss function becomes in this case

$$
\frac{1}{2} \sum_{s}^{n}\left(y_{s}-\beta_{s}\right)^{2}+\lambda_{1} \sum_{s}^{n}\left|\beta_{s}\right|+\lambda_{2} \sum_{(s, t) \in E ; s<t}\left|\beta_{s}-\beta_{t}\right|
$$

which we will refer to as the general Fused Lasso Signal Approximator (FLSA). In the example above, the graph is a 2-D grid.

These examples are only special cases of a more general Fused Lasso model. In this more general form, the Fused Lasso loss function is

$$
L(\mathbf{y}, \mathbf{X}, \boldsymbol{\beta})=\frac{1}{2}(\mathbf{y}-\mathbf{X} \boldsymbol{\beta})^{T}(\mathbf{y}-\mathbf{X} \boldsymbol{\beta})+\lambda_{1} \sum_{i=1}^{p}\left|\beta_{i}\right|+\lambda_{2} \sum_{(i, j) \in E, i<j}\left|\beta_{i}-\beta_{j}\right|
$$


where as above $E$ are the edges in the graph $\mathcal{G}=(V, E)$ with $V=\{1, \ldots, p\}$ representing the variables. Due to space constraints, the algorithm for the general Fused Lasso for matrices $\mathbf{X} \in \mathbb{R}^{n, p}$ where $\operatorname{rank}(X)=p$ and general graphs $\mathcal{G}$ will be given in the Online Supplement in Section 4. For other matrices with $\operatorname{rank}(X)<p$ we do not develop a path algorithm as the solution path w.r.t. $\lambda_{2}$ can have discontinuities in this case. In the following sections, we will present path algorithms for the Fused Lasso Signal Approximator in its special onedimensional and its general form. In Section 2 we will use the special structure of the one-dimensional Fused Lasso Signal Approximator to derive a fast algorithm that calculates the entire solution path with a complexity of $n \log n$. The general FLSA will be treated in Section 3 and is more complicated than the one-dimensional case due to the general structure of the penalty graph $\mathcal{G}$. We prove that our algorithm yields the exact solution and present simulation studies that compare the new algorithms to existing methods. Finally, in Section 4 we will discuss the results and give possible extensions of these algorithms.

\section{One-dimensional Fused Lasso Signal Approximator}

We already used the one-dimensional FLSA in the CGH data example above. Now, in order to develop a path algorithm, we will first have another look at the loss function we seek to minimize:

$$
L(\mathbf{y}, \boldsymbol{\beta})=\frac{1}{2} \sum_{i=1}^{n}\left(y_{i}-\beta_{i}\right)^{2}+\lambda_{1} \sum_{i=1}^{n}\left|\beta_{i}\right|+\lambda_{2} \sum_{i=1}^{n-1}\left|\beta_{i}-\beta_{i+1}\right| .
$$

Due to the simple structure of the loss function, it is possible in this case to obtain the solution for any value of $\left(\lambda_{1}, \lambda_{2}\right)$ by simple soft-thresholding of the solution obtained for $\left(0, \lambda_{2}\right)$. To be more precise, the following theorem holds:

Theorem 1. Assume we have $\mathbf{X}=\mathbf{I}$ and that the solution for $\lambda_{1}=0$ and $\lambda_{2}>0$ is known and denote it by $\boldsymbol{\beta}\left(0, \lambda_{2}\right)$. Then the solution for $\lambda_{1}>0$ is

$$
\beta_{i}\left(\lambda_{1}, \lambda_{2}\right)=\operatorname{sign}\left(\beta_{i}\left(0, \lambda_{2}\right)\right)\left(\left|\beta_{i}\left(0, \lambda_{2}\right)\right|-\lambda_{1}\right)^{+} \quad \text { for } \quad i=1, \ldots, p .
$$

The proof of this theorem is presented in Friedman et al. [2007]. It should be noted that it also holds for the FLSA for arbitrary graphs $\mathcal{G}$, so that it can also be used for the more general FLSA algorithm. For the rest of this section, we assume that $\lambda_{1}=0$. The algorithm presented is a path algorithm that finds the solution for all possible values of $\lambda_{2}$. Any solution for a $\lambda_{1} \neq 0$ can then be obtained by simply soft-thresholding as shown above.

The path algorithm will start by setting $\lambda_{2}=0$ and then increase it until all coefficients $\beta_{i}$ have the same value. For increasing $\lambda_{2}$, neighboring coefficients are forced to be equal to each other. Once this happens, these coefficients are being fused and subsequently treated as a single variable for increasing $\lambda_{2}$. In order to be able to do this, it is important to make sure that these coefficients cannot become unfused again for increasing $\lambda_{2}$. This is in fact the case and will be shown below. Before getting into the details of the algorithm, it is necessary to define some notation.

\subsection{Algorithm}

In order to develop the algorithm for the one-dimensional case, we first have to define what exactly the sets of fused coefficients are. 
Definition 1. Let $F_{i}, i=1, \ldots, n_{F}\left(\lambda_{2}\right)$ be the sets of coefficients at $\lambda_{2}$ that are considered to be fused where $n_{F}\left(\lambda_{2}\right)$ is the number of such sets. In order for these sets to be valid, every set $F_{i}$ has to be of the form $F_{i}=\left\{k \mid l_{i} \leq k \leq u_{i}\right\}$ and the following statements have to hold as well:

- $\cup_{i=1}^{n_{F}\left(\lambda_{2}\right)} F_{i}=\{1, \ldots, n\}$

- $F_{i} \cap F_{j}=\emptyset i \neq j$

- Assuming the $F_{i}$ are ordered, for every $k, l \in F_{i}$ we have $\beta_{k}\left(\lambda_{2}\right)=\beta_{l}\left(\lambda_{2}\right)$ and for $k \in F_{i}, l \in F_{i+1}$ it holds that $\beta_{k}\left(\lambda_{2}\right) \neq \beta_{l}\left(\lambda_{2}\right)$.

For notational convenience, write $\beta_{F_{i}}\left(\lambda_{2}\right)$ for any $\beta_{k}\left(\lambda_{2}\right)$ with $k \in F_{i}$ and also suppress the dependency of $F_{i}$ onto $\lambda_{2}$. Using this definition of fused sets, let us now turn to the algorithm.

For the one-dimensional FLSA, a special result holds that makes the algorithm especially simple and also has been presented in Friedman et al. [2007]. Loosely speaking, it states that if coefficients are fused at $\lambda_{2}^{0}$, then these coefficients will also be fused for any $\lambda_{2}>\lambda_{2}^{0}$. To be more precise

Theorem 2. Let $\beta_{k}\left(\lambda_{2}\right)$ be the optimal solution to the one-dimensional FLSA problem for coefficient $k$ and penalty parameter $\lambda_{2}$. Then if for some $k$ and $\lambda_{2}^{0}$ it holds that $\beta_{k}\left(\lambda_{2}^{0}\right)=$ $\beta_{k+1}\left(\lambda_{2}^{0}\right)$, then for any $\lambda_{2}>\lambda_{2}^{0}$ it holds that $\beta_{k}\left(\lambda_{2}\right)=\beta_{k+1}\left(\lambda_{2}\right)$.

A proof of this theorem is provided in Friedman et al. [2007] and an alternative proof using a different technique is given in the Online Supplement in Section 2.3.

Using this theorem, the algorithm is very simple. First, we need a starting point for the path algorithm and as the optimal solution is known for $\lambda_{2}=0$, which is just $\beta_{k}(0)=y_{k}$ for all $k$, we use it to begin the path. Then the algorithm calculates step by step when two neighboring sets have equal coefficients and merges them. In order to calculate the slope of the coefficient paths for some value of $\lambda_{2}$, we assume that we know $\beta_{k}\left(\lambda_{2}\right)$ for all $k$ as well as the sets of fused variables $F_{i}$. Using this information, we can calculate the derivative of $\beta_{k}\left(\lambda_{2}\right)$ with respect to $\lambda_{2}$ and we will see that these are actually constant, so that the resulting solution path is a piecewise linear function where the breakpoints occur when two set of coefficients are being fused. In order to find $\partial \beta_{k}\left(\lambda_{2}\right) / \partial \lambda_{2}$, define the loss function $L_{F, \lambda_{2}}$ that incorporates the fused sets $F_{i}$. This is done by taking the loss function $L$ in Equation (1) and replacing $\beta_{k}$ by $\beta_{F_{i}}$ for $k \in F_{i}$. This constrained loss function is then

$$
L_{F, \lambda_{2}}(\mathbf{y}, \boldsymbol{\beta})=\frac{1}{2} \sum_{i=1}^{n_{F}\left(\lambda_{2}\right)}\left(\sum_{j \in F_{i}}\left(y_{j}-\beta_{F_{i}}\right)^{2}\right)+\lambda_{2} \sum_{i=1}^{n_{F}\left(\lambda_{2}\right)-1}\left|\beta_{F_{i}}-\beta_{F_{i+1}}\right| .
$$

Due to the assumptions on the sets $F$, the constrained loss function $L_{F, \lambda_{2}}$ is always differentiable with respect to $\beta_{F_{i}}$ unless two coefficients are just being fused (which only happens if $\beta_{F_{i}}=\beta_{F_{i+1}}$ for some $i$ ). Assuming that $\beta_{F_{i}}$ is optimal, the derivative of $L_{F, \lambda_{2}}$ has to be 0 and we get

$$
\begin{aligned}
\frac{\partial L_{F, \lambda_{2}}}{\partial \beta_{F_{i}}} & =\left|F_{i}\right| \beta_{F_{i}}-\sum_{j \in F_{i}} y_{j}+\lambda_{2} \operatorname{sign}\left(\beta_{F_{i}}-\beta_{F_{i-1}}\right)+ \\
& +\lambda_{2} \operatorname{sign}\left(\beta_{F_{i}}-\beta_{F_{i+1}}\right)=0 \quad \text { for } \quad i=1, \ldots, n_{F}\left(\lambda_{2}\right)
\end{aligned}
$$




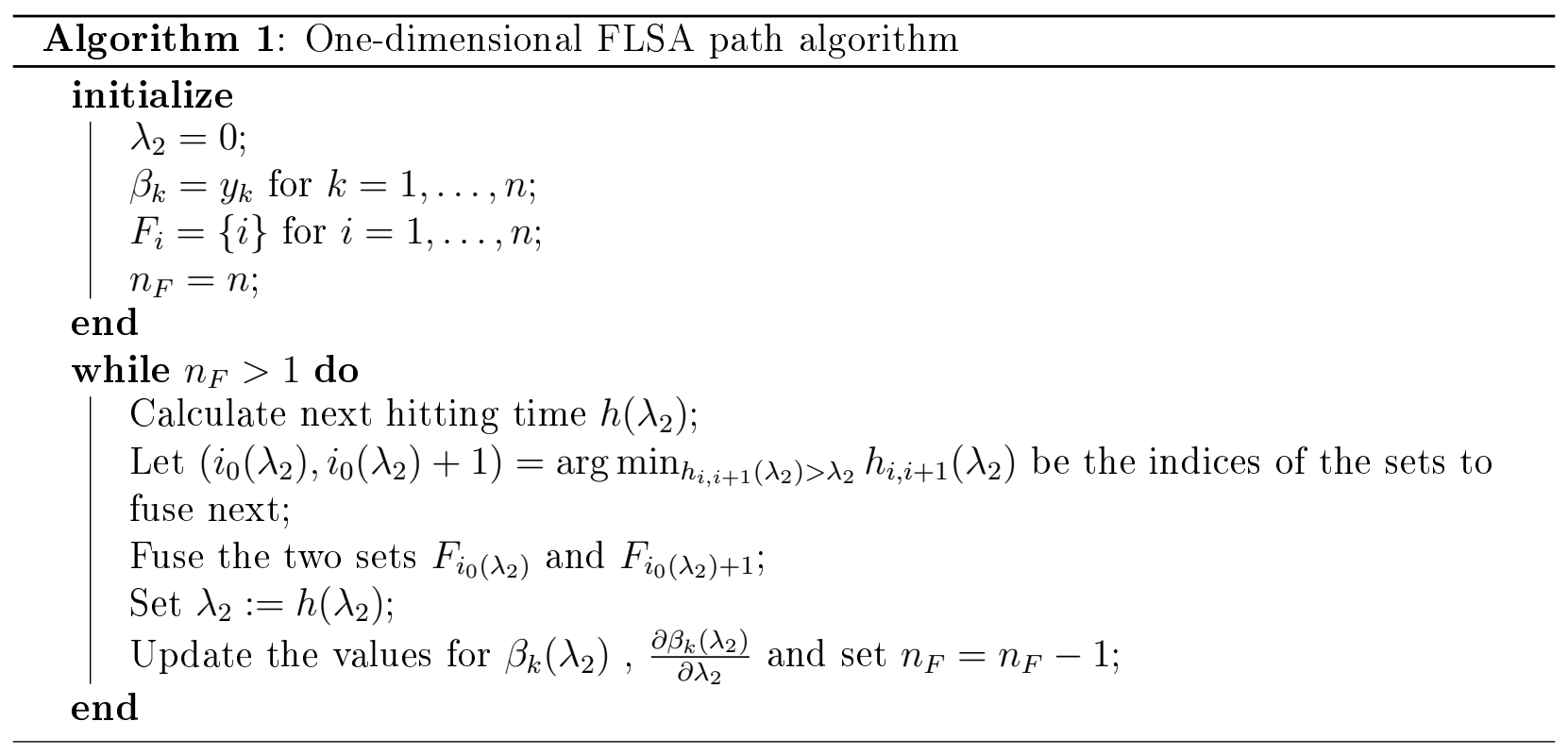

where we set $\operatorname{sign}\left(\beta_{F_{1}}-\beta_{F_{0}}\right)=0$ and $\operatorname{sign}\left(\beta_{F_{n_{F}\left(\lambda_{2}\right)}}-\beta_{F_{n_{F}\left(\lambda_{2}\right)+1}}\right)=0$. Now, taking derivatives with respect to $\lambda_{2}$ in these equations gives the results

$$
\frac{\partial \beta_{F_{i}}}{\partial \lambda_{2}}=-\frac{1}{\left|F_{i}\right|}\left(\operatorname{sign}\left(\beta_{F_{i}}-\beta_{F_{i-1}}\right)+\operatorname{sign}\left(\beta_{F_{i}}-\beta_{F_{i+1}}\right)\right) \quad \text { for } \quad i=1, \ldots, n_{F}\left(\lambda_{2}\right)
$$

As we already mentioned above, these are constant as long as the sets of fused coefficients do not change. By Theorem 2, we know that the only way to change the sets of fused coefficients is to merge two sets as they can never split for increasing $\lambda_{2}$. Therefore, the solution path is piecewise linear and for increasing $\lambda_{2}$ the breakpoint occurs when two groups fuse. Thus, it is easy to calculate the next breakpoint, which occurs when neighboring sets have the same coefficients. In order to do this, define

$$
h_{i, i+1}\left(\lambda_{2}\right)=\frac{\beta_{F_{i}}\left(\lambda_{2}\right)-\beta_{F_{i+1}}\left(\lambda_{2}\right)}{\frac{\partial \beta_{F_{i+1}}}{\partial \lambda_{2}}-\frac{\partial \beta_{F_{i}}}{\partial \lambda_{2}}}+\lambda_{2} \quad \text { for } \quad i=1, \ldots, n_{F}\left(\lambda_{2}\right)-1
$$

which is the value for $\lambda_{2}$ at which the coefficients of the sets $F_{i}$ and $F_{i+1}$ have the same value and can be fused, assuming that no other coefficients become fused before that. If $h_{i, i+1}\left(\lambda_{2}\right)<\lambda_{2}$, these values are being ignored as the two groups $F_{i}$ and $F_{i+1}$ are actually moving apart for increasing $\lambda_{2}$. The next value at which coefficients are fused is therefore the hitting time

$$
h\left(\lambda_{2}\right)=\min _{h_{i, i+1}>\lambda_{2}} h_{i, i+1}\left(\lambda_{2}\right) .
$$

As we are taking the minimum, it is only defined if there is at least one $h_{i, i+1}>\lambda_{2}$. From equation (1) with $\lambda_{1}=0$ we can easily see that for $\lambda_{2} \rightarrow \infty$ the solution is $\beta_{k}=\frac{1}{n} \sum_{l=1}^{n} y_{l}$ for all $k$, thus only one group exists for large $\lambda_{2}$. Therefore, if $n_{F}\left(\lambda_{2}\right) \geq 2$, then there exists an $h_{i, i+1}>\lambda_{2}$ and therefore $h\left(\lambda_{2}\right)$ is defined. Based on these results, we can now write out the details of the algorithm that provides the entire solution path and they can be found in Algorithm 1.

As we will show below, this algorithm only requires a low number of computational steps 


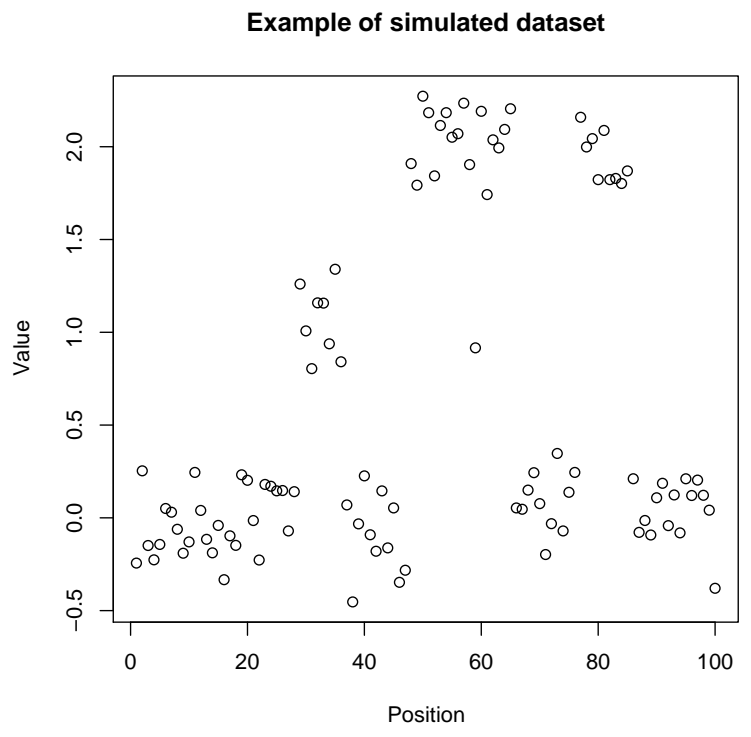

Figure 3: An example of a simulated dataset with size $n=100$ for the one-dimensional FLSA.

and is of complexity $n \log (n)$. Of course, apart from the computational complexity, it is also important to be able to save the results in an efficient manner. This can be done with memory usage on the order $O(n)$. A more detailed analysis can be found in the Online Supplement in Section 2.

\subsection{Speed comparison}

In order to evaluate the speed of our new algorithm, we want to compare it to other methods that have been published before. The first alternative we also use is the component-wise algorithm presented in Friedman et al. [2007]. The second is based on the general convex solver $C V X$, a package for specifying and solving convex problems (see Grant and Boyd [2008a,b]). CVX is very easy to use and flexible, which is why we chose it, despite the disadvantage that it cannot be used with a warm start.

As datasets of a wide range of sizes are needed, the speed comparisons will be performed on simulated data. The simulated dataset consists of datapoints with values of 0,1 and 2. Roughly $20 \%$ of datapoints will have value 1 and $20 \%$ value 2 . An example plot of a simulated dataset of size $n=100$ can be seen in Figure 3.

When calculating the solution, our new path algorithm and the competing methods take somewhat different approaches. Our algorithm calculates the whole solution path whereas the competing method calculate the solution only for a prespecified list of $\lambda_{2}$ values. In order to make the two approaches comparable we measure the time each algorithm takes to find the solutions for 50 values of $\lambda_{2}$ which are equally spaced between 0 and 1 . The results of the comparison can be found in Table 1.

As it can be seen, the path algorithm is consistently faster than the component-wise optimization algorithm for all but the largest problems. They are also both much faster than the general convex solver CVX. In addition to this, the path algorithm also returns an object that stores the complete solution path in a compact form and can be used to extract 


\begin{tabular}{|c|c|c|c|c|c|c|}
\hline $\mathrm{n}$ & $10^{2}$ & $10^{3}$ & $10^{4}$ & $10^{5}$ & $10^{6}$ & $10^{7}$ \\
\hline \hline CVX & 17.1 & 30.2 & 210 & 3600 & $>5$ hours & $>5$ hours \\
\hline Component-wise Alg. & 0.071 & 0.081 & 0.24 & 1.1 & 10 & 98 \\
\hline Path Alg. & 0.0006 & 0.003 & 0.030 & 0.52 & 7.8 & 108 \\
\hline
\end{tabular}

Table 1: Time in seconds for a 1-dimensional FLSA problem of size $n$. All three algorithms calculate the solution for 50 equally spaced values of $\lambda_{2}$ from 0 to 1 . Results averaged over 10 simulations.

solutions for additional values of $\lambda_{2}$ very quickly.

After deriving the path algorithm for the one-dimensional Fused Lasso Signal Approximator, we want to generalize the algorithm to the case of the general Fused Lasso Signal Approximator. The most important difference to the previous algorithm is that a set of fused coefficients can also break into several sets for increasing values of $\lambda_{2}$. We will get into more detail in the next section.

\section{General Fused Lasso Signal Approximator}

In the introduction we have already seen an example where a more general penalty structure than in the one-dimensional FLSA can be very useful for reconstructing a noisy image. However, we do not need to restrict our attention to a two-dimensional grid. In this section, we will present an algorithm that finds the solution for the FLSA problem with an arbitrary graph $\mathcal{G}=(V, E)$ (with set of vertices $V=\{1, \ldots, n\}$ and edges $E$ ) specifying the structure of the penalty parameter on differences. The loss function in this case is

$$
L(\mathbf{y}, \boldsymbol{\beta})=\frac{1}{2} \sum_{i=1}^{n}\left(y_{i}-\beta_{i}\right)^{2}+\lambda_{1} \sum_{i=1}^{p}\left|\beta_{i}\right|+\lambda_{2} \sum_{(i, j) \in E, i<j}\left|\beta_{i}-\beta_{j}\right|
$$

so that we penalize $\left|\beta_{i}-\beta_{j}\right|$ for every edge $(i, j) \in E$. The condition $i<j$ only makes sure that we penalize $\left|\beta_{i}-\beta_{j}\right|$ only once as the edges in the graph are assumed to be undirected. As in the previous section, we can use the soft-thresholding theorem (see Theorem 1) and it is therefore possible to set $\lambda_{1}=0$ and find a solution path for $\lambda_{2}$ and later obtain any solution for $\lambda_{1}>0$ by soft-thresholding. The algorithm for this more general case is conceptually similar to the one presented for the one-dimensional case. However, unlike in this simpler setup, for the more general penalty structure it is not guaranteed that a group of coefficients that is fused for value $\lambda_{2}=\lambda_{2}^{0}$ will remain fused for $\lambda_{2}>\lambda_{2}^{0}$, but instead fused groups may break up for increasing $\lambda_{2}$. The main adjustment to deal with this problem will be to introduce a method to determine for which value of $\lambda_{2}$ a group of fused variables will break up.

In the following subsections, we will first make some necessary adjustments to the definition of sets of fused variables and given these sets, we calculate $\partial \beta_{F_{i}} / \partial \lambda_{2}$. Next, we give the conditions under which a set of fused coefficients breaks up into two smaller sets and present a method on how to calculate critical values of $\lambda_{2}$ for which this could happen. After incorporating this into the final algorithm, we present an approximate version of our method that is faster on large dataset by sacrificing some precision. Finally, we use our new algorithm on simulated data and compare its speed and accuracy to the other methods we have 
already used for the one-dimensional FLSA. Now, let us first make the necessary changes to the definition of fused coefficients to account for the general graph structure $\mathcal{G}$.

\subsection{Sets of fused variables}

In the case of the one-dimensional FLSA before, we already specified certain conditions for the sets of fused variables $F_{i}$ in Definition 1 . Here, due to the more complicated structure of the graph $\mathcal{G}$, we have to restate the condition that any set has to be an interval. For general graphs, the condition is instead that any set of fused variables has to be connected. For the definition and also in the following sections, assume that we know the minimizer of the loss function for penalty parameter $\lambda_{2}$ and denote it by $\beta_{F_{i}}\left(\lambda_{2}\right)$. Then the definition of a valid set of fused variables is:

Definition 2. Let $n_{F}\left(\lambda_{2}^{0}\right)$ be the number of sets of fused variables for penalty parameter $\lambda_{2}^{0}$. Then for the sets $F_{i}, i=1, \ldots, n_{F}\left(\lambda_{2}^{0}\right)$ to be valid, the following conditions have to hold:

1. $\cup_{i=1}^{n_{F}\left(\lambda_{2}\right)} F_{i}=\{1, \ldots, n\}$

2. $F_{i} \cap F_{j}=\emptyset i \neq j$

3. If $k, l \in F_{i}$ then $\beta_{k}\left(\lambda_{2}^{0}\right)=\beta_{l}\left(\lambda_{2}^{0}\right)$ and if $k \in F_{i}, l \in F_{j}, i \neq j$ and $F_{i}$ and $F_{j}$ have a connecting edge, then $\beta_{k}\left(\lambda_{2}\right) \neq \beta_{l}\left(\lambda_{2}\right)$ for all penalty parameters $\lambda_{2} \in\left(\lambda_{2}^{0}, \lambda_{2}^{0}+\varepsilon\right)$ for some $\varepsilon>0$.

4. If $k, l \in F_{i}$ then $k$ and $l$ are connected in $\mathcal{G}$ by only going over nodes in $F_{i}$, i.e. $k, l$ are connected in $\left.\mathcal{G}\right|_{F_{i}}$, the subgraph of $\mathcal{G}$ induced by $F_{i}$.

Compared to the previous version of this definition, the third and fourth condition have been adapted. The third condition now reflects that sets can be split up for increasing $\lambda_{2}$ and the last one is equivalent to the requirement that $F_{i}$ is an interval in the one-dimensional FLSA case.

Now, assuming that the sets of variables $F_{i}$ that are fused are given, we will determine the slope of the optimal $\beta_{F_{i}}$ with respect to $\lambda_{2}$. In order to do this, we will incorporate the sets of fused coefficients into the loss function (2). Setting $\beta_{k}=\beta_{F_{i}}$ for all $k \in F_{i}$, the loss function becomes

$$
\begin{aligned}
L_{F, \lambda_{2}}(\mathbf{y}, \boldsymbol{\beta}) & =\sum_{i=1}^{n_{F}\left(\lambda_{2}\right)}\left(\sum_{j \in F_{i}}\left(y_{j}-\beta_{F_{i}}\right)^{2}\right)+ \\
& +\lambda_{2} \sum_{i<j}\left|\left\{(k, l) \in E: k \in F_{i}, l \in F_{j}\right\}\right|\left|\beta_{F_{i}}-\beta_{F_{j}}\right| .
\end{aligned}
$$

Here, note that by definition of the sets $F_{i}$, we have that $\beta_{F_{i}}\left(\lambda_{2}\right) \neq \beta_{F_{j}}\left(\lambda_{2}\right)$ for $j \neq i$ (except for a finite number of $\lambda_{2}$ for which sets are fused or split) and therefore the loss function is differentiable with respect to $\beta_{F_{i}}$ at the solution $\beta_{F_{i}}\left(\lambda_{2}\right)$. Thus, at $\beta_{F_{i}}\left(\lambda_{2}\right)$, the 
derivative of $L_{F, \lambda_{2}}(\mathbf{y}, \boldsymbol{\beta})$ with respect to $\beta_{F_{i}}$ is 0 , that is

$$
\begin{aligned}
\frac{\partial L_{F, \lambda_{2}}(\mathbf{y}, \boldsymbol{\beta})}{\partial \beta_{F_{i}}} & =\left|F_{i}\right| \beta_{F_{i}}-\sum_{j \in F_{i}} y_{j}+ \\
& +\lambda_{2} \sum_{j \neq i}\left|\left\{(k, l) \in E: k \in F_{i}, l \in F_{j}\right\}\right| \operatorname{sign}\left(\beta_{F_{i}}-\beta_{F_{j}}\right)=0
\end{aligned}
$$

for the solutions $\beta_{F_{i}}\left(\lambda_{2}\right)$. By taking the derivative w.r.t. $\lambda_{2}$ and noting that for small changes of $\lambda_{2}$, the sign of $\beta_{F_{i}}-\beta_{F_{j}}$ does not change, it is possible to determine $\partial \beta_{F_{i}}\left(\lambda_{2}\right) / \partial \lambda_{2}$ as

$$
\frac{\partial \beta_{F_{i}}\left(\lambda_{2}\right)}{\partial \lambda_{2}}=-\frac{\sum_{j \neq i}\left|\left\{(k, l) \in E: k \in F_{i}, l \in F_{j}\right\}\right| \operatorname{sign}\left(\beta_{F_{i}}-\beta_{F_{j}}\right)}{\left|F_{i}\right|}
$$

which is constant as long as the $F_{i}$ do not change. Therefore, the solution $\beta_{F_{i}}\left(\lambda_{2}\right)$ is a piecewise linear function again. At the breakpoints of the solution path, the sets of fused variables change. As we will see in more detail later, there are 2 things that can happen:

- $\beta_{F_{i}}\left(\lambda_{2}\right)=\beta_{F_{j}}\left(\lambda_{2}\right)$ for some $i \neq j$ with $F_{i}$ and $F_{j}$ connected, which violates condition 3 of Definition 2. In this case, fuse sets $F_{i}$ and $F_{j}$.

- A set $F_{i}$ has to be broken up into 2 smaller subsets. A way to determine when this has to happen is presented below.

Once the sets have been updated, the solution path is again linear so that the whole solution path for $\lambda_{2}$ can be obtained by updating the sets of fused coefficients at the right values of $\lambda_{2}$. Now we will take a closer look at the conditions under which we split a set of fused variables.

\subsection{Splitting and fusing sets of variables}

In order to see when it is necessary to split sets of variables, we have to see when the solution $\beta_{F_{i}}\left(\lambda_{2}\right)$ and its derivatives obtained from the constrained loss function $L_{F, \lambda_{2}}$ from Equation (3) is also optimal for the unconstrained loss $L_{\lambda_{2}}$ in Equation (2). For this, we will look at the subgradient equations of $L_{\lambda_{2}}$. An overview of subgradients can be found in Bertsekas [1999].

\subsubsection{Subgradient equations}

As $L_{\lambda_{2}}$ is not differentiable everywhere, it is convenient to use subgradients instead of the usual derivatives. For the subgradients, a necessary and sufficient condition for $\beta_{k}$ to be optimal is that

$$
\frac{\partial L_{\lambda_{2}}(\mathbf{y}, \boldsymbol{\beta})}{\partial \beta_{k}}=\beta_{k}-y_{k}+\lambda_{2} \sum_{(k, l) \in E} t_{k l}=0 \quad \text { for } \quad k=1, \ldots, n
$$

where $t_{k l}=\operatorname{sign}\left(\beta_{k}-\beta_{l}\right)$ for $\beta_{k} \neq \beta_{l}, t_{k l} \in[-1,1]$ for $\beta_{k}=\beta_{l}$. For the case $\beta_{k}=\beta_{l}$ it is also enforced that $t_{k l}=-t_{l k}$ (which is trivially true in the $\beta_{k} \neq \beta_{l}$ case). Given a grouping $F_{i}$, 
these equations can be written slightly differently. With $k \in F_{i}$, let

$$
\frac{\partial L_{\lambda_{2}}(\mathbf{y}, \boldsymbol{\beta})}{\partial \beta_{k}}=\beta_{k}-y_{k}+\lambda_{2} \sum_{i \neq j} \sum_{(k, l) \in E: l \in F_{j}} t_{k l}+\lambda_{2} \sum_{(k, l) \in E: l \in F_{i}} t_{k l}=0 \text { for } k=1, \ldots, n
$$

where we grouped the $t_{k l}$ for which $k, l \in F_{i}$. Assuming that $\beta_{F_{i}}\left(\lambda_{2}\right)$ is a minimizer of the unconstrained loss function, there exist $t_{k l}\left(\lambda_{2}\right)$ such that the subgradient equations hold. Writing $\tau_{k l}\left(\lambda_{2}\right)=\lambda_{2} t_{k l}\left(\lambda_{2}\right)$ and taking the derivative w.r.t. $\lambda_{2}$ in the subgradient equations, we get

$$
\frac{\partial \beta_{k}}{\partial \lambda_{2}}+\sum_{i \neq j} \sum_{(k, l) \in E: l \in F_{j}} t_{k l}+\sum_{(k, l) \in E: l \in F_{i}} \frac{\partial \tau_{k l}}{\partial \lambda_{2}}=0 \text { for } k=1, \ldots, n .
$$

where we exploit the fact that $t_{k l}= \pm 1$ is constant for $k \in F_{i}$ and $l \in F_{j}$. Note that there are not necessarily unique values for $\partial \tau_{k l} / \partial \lambda_{2}$ such that these equations hold and there may be an infinite number of possible solutions, any of which will serve our purpose. Also note that from above we know that as long as the set $F_{i}$ stays fixed, $\partial \beta_{k} / \partial \lambda_{2}$ is constant for $k \in F_{i}$. This is the case because $t_{k l}$ for $k \in F_{j}$ and $l \in F_{i}$ with $i \neq j$ is defined as $t_{k l}=\operatorname{sign}\left(\beta_{k}-\beta_{l}\right)$ and stays constant as long as the order of $\beta_{k}$ and $\beta_{l}$ does not change. However, if the order changes, then by Definition 2 the set $F_{i}$ has to change as well. Therefore, the equation above stays the same as long as $F_{i}$ is fixed. Therefore, the $\partial \tau_{k l} / \partial \lambda_{2}$ only depend on the groups and not on $\lambda_{2}$. However, apart from Equation (6), it also has to hold that $\tau_{k l}\left(\lambda_{2}\right) \in\left[-\lambda_{2}, \lambda_{2}\right]$, as the condition $t_{k l} \in[-1,1]$ has to hold. Thus, when we keep the groups $F_{i}$ fixed, it will not always be possible to find $\partial \tau_{k l} / \partial \lambda_{2}$ such that for increasing $\lambda_{2}$ these conditions still hold. As $\partial \tau_{k l} / \partial \lambda_{2}$ is constant, the $\tau_{k l}$ are piecewise linear and the $t_{k l}$ are continuous in $\lambda_{2}$. Then there are two ways in which the subgradient conditions can fail:

1. There is a group $F_{i}$ for which for increasing $\lambda_{2}$, the condition $-1 \leq t_{k l} \leq 1$ for all $k, l \in F_{i}$ cannot be satisfied anymore. In this case, the group has to be split into two smaller subgroups. How to decide when this is the case and how to identify the two new subgroups will be treated below.

2. There are two groups $F_{i}$ and $F_{j}$ for which there exists $k \in F_{i}$ and $l \in F_{j}$ with $(k, l) \in E$, i.e. $F_{i}$ and $F_{j}$ have at least one edge connecting them. For these two groups, we have that $t_{k l}= \pm 1$ does not hold anymore for all $k \in F_{i}$ and $l \in F_{j}$. In this case, the groups $F_{i}$ and $F_{j}$ have to be fused.

It is very easy to detect when the second case above occurs, i.e., when two groups have to be fused. If we have two groups $F_{i}$ and $F_{j}$ that are directly connected to each other by an edge, then they will be fused at $\lambda_{2}^{0}$ if $\beta_{F_{i}}\left(\lambda_{2}^{0}\right)=\beta_{F_{j}}\left(\lambda_{2}^{0}\right)$ and $\beta_{F_{i}}\left(\lambda_{2}\right) \neq \beta_{F_{j}}\left(\lambda_{2}\right)$ for some interval $\lambda_{2} \in\left(\lambda_{2}^{0}-\varepsilon, \lambda_{2}^{0}\right)$, i.e. groups $F_{i}$ and $F_{j}$ "hit" each other at $\lambda_{2}^{0}$. Detecting when a group has to be broken into two smaller groups is harder and we will discuss it in the following subsection.

\subsubsection{The maximum flow problem}

In order to decide when to split variables, it is necessary to find solutions for $t_{k l}\left(\lambda_{2}\right)$ (or equivalently $\tau_{k l}$ ) in Equation (6). For $k$ and $l$ that are not in the same group, this will be easy as then $t_{k l}=\operatorname{sign}\left(\beta_{F_{i}}-\beta_{F_{j}}\right)$ for $k \in F_{i}$ and $l \in F_{j}$. For $k$ and $l$ in the same group, i.e. $k, l \in F_{i}$, we will see that $\tau_{k l}$ is an affine function of $\lambda_{2}$ and the slope can be calculated by 
solving a maximum flow problem in graph theory. The same calculations can also be used to identify when a group has to be split by (more on this later). As maximum flow problems are a well studied area, fast algorithms for this problem such as the push-relabel algorithm, among others, exist and can be used here (see Cormen et al. [2001]). Before going into more details, assume that for penalty parameter $\lambda_{2}$, we know $\tau_{k l}\left(\lambda_{2}\right)$ for $k, l \in F_{i}, i=1, \ldots, n_{F}\left(\lambda_{2}\right)$ that solve the subgradient Equation (6). Now, for notational convenience, define

$$
p_{k}=-\sum_{i \neq j} \sum_{(k, l) \in E: l \in F_{j}} t_{k l}-\frac{\partial \beta_{F_{i}}\left(\lambda_{2}\right)}{\partial \lambda_{2}}
$$

for $k \in F_{i}$ and call it the push on node $k$ as it measures the influence other variables in neighboring groups that are connected to $k$ have on node $k$. Using these definitions, Equation (6) can be written as

$$
\sum_{(k, l) \in E: l \in F_{i}} \frac{\partial \tau_{k l}}{\partial \lambda_{2}}=p_{k} \quad \text { for } \quad k=1, \ldots, n .
$$

For each of these equations, we can see that they only involve variables that belong the same groups, i.e. if $k \in F_{i}$, then all $l$ used for the variables $\tau_{k l}$ are also in $F_{i}$ and thus these $n$ equations are separable according to the groups $F_{i}$. Therefore, for each of the groups $F_{i}$, we will solve a separate maximum flow problem to find $\partial \tau_{k l} / \partial \lambda_{2}$ and determine if it is necessary to split the group.

For any maximum flow problem, we need to specify the underlying graph which consists of vertices, edges and capacities on each edge in both directions. In order to do this, we first define the graph $\mathcal{G}_{i}=\left.\mathcal{G}\right|_{F_{i}}$, which is the graph $\mathcal{G}$ restricted to the nodes in the set $F_{i}$. Now let $\tilde{\mathcal{G}}_{i}=\left(\tilde{V}_{i}, \tilde{E}_{i}, \tilde{C}_{i}\right)$ be the vertices, edges and capacities of the $i$-th problem. For these, we define:

Vertices: To each of the subgraphs $\mathcal{G}_{i}$, we add an artificial source node $r$ and sink node $s$, such that $\tilde{V}_{i}=V_{i} \cup\{r, s\}$.

Edges: For the edges $\tilde{E}_{i}$ we use all the edges in $E_{i}$ and will add additional edges connecting each of the nodes in $V_{i}$ to either the source or the sink. In order to motivate which nodes will be connected to which, note that at the end, we will set $\partial \tau_{k l} / \partial \lambda_{2}=f_{k l}$ for the maximal flow $f_{k l}$ from node $k$ to node $l$. As the flow through every node (except for the source and sink) has to be 0, a node has an edge with the source if the RHS of equation (7) is greater than 0 and an edge with the sink if it is less than 0 . Thus

$$
\tilde{E}_{i}=E_{i} \cup\left\{(r, l): p_{l}>0\right\} \cup\left\{(k, s): p_{k}<0\right\}
$$

As usual, all the edges are undirected.

Capacities: Of course, the capacities on the edges have to be defined as well and we will define them for each direction separately. As we will set $\partial \tau_{k l} / \partial \lambda_{2}=f_{k l}$ for $k, l \in F_{i}$, the flows have to be constrained such that $\tau_{k l}\left(\lambda_{2}\right)$ stays within the interval $\left[-\lambda_{2}, \lambda_{2}\right]$. This has to hold for all edges in $E_{i}$. For the edges to the source or the sink, the corresponding absolute value on the RHS of Equation (7) will be used. This way, with $\partial \tau_{k l} / \partial \lambda_{2}=f_{k l}$, Equation (7) will hold if and only if all edges coming from the source and all edges 
going to the sink are at full capacity.

In order to ensure that $\tau_{k l}\left(\lambda_{2}\right) \in\left[-\lambda_{2}, \lambda_{2}\right]$, we have the restrictions

$$
\frac{\partial \tau_{k l}}{\partial \lambda_{2}} \in \begin{cases}(-\infty, \infty) & \text { if } \tau_{k l} \in\left(-\lambda_{2}, \lambda_{2}\right) \\ (-\infty, 1] & \text { if } \tau_{k l}=\lambda_{2} \\ {[-1, \infty)} & \text { if } \tau_{k l}=-\lambda_{2}\end{cases}
$$

Correspondingly, we set for $k, l \in F_{i}$

$$
\left(c_{k l}, c_{l k}\right)= \begin{cases}(-\infty, \infty) & \text { if } \tau_{k l} \in\left(-\lambda_{2}, \lambda_{2}\right) \\ (1, \infty) & \text { if } \tau_{k l}=\lambda_{2} \\ (\infty, 1) & \text { if } \tau_{k l}=-\lambda_{2} .\end{cases}
$$

Now it only remains to define the capacities on the edges coming from the source or going to the sink. First, for the edges from the source $r$, i.e. all $l$ for which $(r, l) \in \tilde{E}_{i}$,

$$
\left(c_{r l}, c_{l r}\right)=\left(p_{l}, 0\right)
$$

and correspondingly for the edges to the sink set for all $k$ with $(k, s) \in \tilde{E}_{i}$,

$$
\left(c_{k s}, c_{s k}\right)=\left(-p_{k}, 0\right) \text {. }
$$

Using all this set $\tilde{C}_{i}=\left\{c_{k l}: k, l \in \tilde{V}_{i}\right\}$.

Here it is interesting to note that

$$
\sum_{k \in F_{i}} p_{k}=0
$$

which is easy to see by summing up Eequations (6), the definition of $p_{k}$ and that $t_{k l}=-t_{l k}$. Therefore, the sum of all capacities going out of the source is equal to the sum of all capacities going into the sink and thus a flow that is maximal for all source edges is also maximal for all sink edges.

Now that we have defined the maximal flow problem, we will not go into any detail of how to solve it and just refer to the literature that we have already mentioned above. In the following, the solution to the flow problem will be referred to as $f_{k l}$, which is the flow from node $k$ to node $l$, assuming that $k, l \in \tilde{E}_{i}$. Using this result we will now show that the solution path is piecewise-linear. The next theorem guarantees that for an interval, the solution for $\beta_{k}\left(\lambda_{2}\right)$ and $\tau_{k l}\left(\lambda_{2}\right)$ are affine and have the slope as stated above.

Theorem 3. For some $\lambda_{2}^{0}$, let $F_{1}, \ldots, F_{n_{F}\left(\lambda_{2}^{0}\right)}$ be a valid grouping of the variables. Let $\tilde{\mathcal{G}}_{i}$ be the with $F_{i}$ associated maximum-flow graph as defined above. Also let $\beta_{k}\left(\lambda_{2}^{0}\right)$ and $\tau_{k l}\left(\lambda_{2}^{0}\right)$ be a solution to the FLSA problem for penalty parameter $\lambda_{2}=\lambda_{2}^{0}$. If $\tilde{\mathcal{G}}_{i}$ has a maximum flow for which all flows coming from the source are at maximum capacity (i.e. $f_{r l}=c_{r l}$ for all $\left.(r, l) \in \tilde{E}_{i}\right)$, and $\frac{\partial \beta_{F_{i}}}{\partial \lambda_{2}}$ is as defined in equation (4), then there exists some $\Delta>0$ such that for any $\lambda_{2} \in\left[\lambda_{2}^{0}, \lambda_{2}^{0}+\Delta\right]$, the solution to the FLSA problem is given by

$$
\beta_{k}\left(\lambda_{2}\right)=\beta_{k}\left(\lambda_{2}^{0}\right)+\frac{\partial \beta_{F_{i}}}{\partial \lambda_{2}}\left(\lambda_{2}^{0}\right) \cdot\left(\lambda_{2}-\lambda_{2}^{0}\right) \quad \text { for } \quad k \in F_{i}
$$


and

$$
\tau_{k l}\left(\lambda_{2}\right)= \begin{cases}\tau_{k l}\left(\lambda_{2}^{0}\right)+f_{k l}\left(\lambda_{2}-\lambda_{2}^{0}\right) & \text { for } k, l \in F_{i} \\ \operatorname{sign}\left(\tau_{k l}\left(\lambda_{2}^{0}\right)\right) \lambda_{2} & \text { otherwise. }\end{cases}
$$

The proof of this theorem can be found in the Online Supplement in Section 3. The only item in the previous proof that we haven't specified so far is the length of the interval $\Delta$, for which the solution will be linear as described. There are two things that can occur, that would violate the assumptions of Theorem 3 . First, two sets of variables $F_{i}$ and $F_{j}$ that have a connecting edge have $\beta_{F_{i}}=\beta_{F_{j}}$ and therefore, the grouping is not valid anymore. In this case, the two sets have to be merged. Second, for some group $F_{i}$, the maximum flow problem is not at maximal capacity for all source nodes. Then, this group has to be split. However, before proving that these operations yield a valid grouping for which the assumption of Theorem 3 hold, we will determine $\Delta$.

\subsubsection{The hitting time $h$ and splitting time $v$}

In order to find $\Delta$, we will first determine the smallest value $\lambda_{2}>\lambda_{2}^{0}$ where two neighboring groups have the same coefficient. Next, we will determine the smallest $\lambda_{2}>\lambda_{2}^{0}$ such that the conditions on $\tau_{k l}$ are violated. $\lambda_{2}^{0}+\Delta$ is then the smallest of these two values.

Start by assuming that for penalty parameter $\lambda_{2}^{0}$ we have a valid grouping $F_{1}, \ldots, F_{n_{F}\left(\lambda_{2}^{0}\right)}$ and solutions $\beta_{k}\left(\lambda_{2}^{0}\right)$ as well as $\tau_{k l}\left(\lambda_{2}^{0}\right)$. Given this, it is easy to calculate when two sets that are connected by an edge hit. For this, let the hitting time of groups $i$ and $j$ at $\lambda_{2}^{0}$ be

$$
h_{i j}\left(\lambda_{2}^{0}\right)= \begin{cases}\left(\beta_{F_{i}}-\beta_{F_{j}}\right) /\left(\frac{\partial \beta_{F_{j}}}{\partial \lambda_{2}}-\frac{\partial \beta_{F_{i}}}{\partial \lambda_{2}}\right)+\lambda_{2}^{0} & \text { if } \exists k \in F_{i}, l \in F_{j} \text { with }(k, l) \in E \\ \infty & \text { otherwise. }\end{cases}
$$

If $h_{i j}<\lambda_{2}^{0}$, then given the current slopes, these groups will not meet for $\lambda_{2}>\lambda_{2}^{0}$. If $h_{i j}=\lambda_{2}^{0}$, then $\beta_{F_{i}}\left(\lambda_{2}^{0}\right)=\beta_{F_{j}}\left(\lambda_{2}^{0}\right)$. However, as we assumed that this is a valid grouping, from the definition we get that for some $\varepsilon>0$, for any $\lambda_{2} \in\left(\lambda_{2}^{0}, \lambda_{2}^{0}+\varepsilon\right)$ we have $\beta_{F_{i}}\left(\lambda_{2}\right) \neq \beta_{F_{j}}\left(\lambda_{2}\right)$ and as the trajectories are piecewise affine, the groups $F_{i}$ and $F_{j}$ move apart. Therefore, defining

$$
h\left(\lambda_{2}^{0}\right)=\min _{h_{i j}>\lambda_{2}^{0}} h_{i j}\left(\lambda_{2}^{0}\right)
$$

we have that two groups will hit at $h$ but not before. Therefore, the grouping remains valid for at least an interval $h-\lambda_{2}^{0}$.

Now let us look at the maximum flow problem and how long the interval can be such that for all $\tau_{k l}\left(\lambda_{2}\right) \in\left[-\lambda_{2}, \lambda_{2}\right]$. Then, given the flows $f_{k l}$, define the violation time of the constraint on $\tau_{k l}$ as

$$
v_{k l}\left(\lambda_{2}^{0}\right)= \begin{cases}\frac{\left|\operatorname{sign}\left(f_{k l}\right) \lambda_{2}^{0}-\tau_{k l}\left(\lambda_{2}^{0}\right)\right|}{\left|f_{k l}\right|-1}+\lambda_{2}^{0} & \text { if }\left|f_{k l}\right|>1 \\ \infty & \text { otherwise }\end{cases}
$$

and set

$$
v\left(\lambda_{2}^{0}\right)=\min v_{k l}\left(\lambda_{2}^{0}\right) .
$$

Then given the behavior of the $\tau_{k l}$ described in Theorem 3, we have that $\tau_{k l}\left(\lambda_{2}\right) \in\left[-\lambda_{2}, \lambda_{2}\right]$ 
for $\lambda_{2} \in\left[\lambda_{2}^{0}, v\right]$. Furthermore, for $\lambda_{2}>\lambda_{2}^{0}+v$, at least one of the constraints would be violated. However, note that this does not necessarily mean that the group has to be split. It may just be necessary to find a new maximal flow that satisfies the constraints. Using the hitting and violation time, we can set

$$
\Delta=\min \left(h\left(\lambda_{2}^{0}\right), v\left(\lambda_{2}^{0}\right)\right)-\lambda_{2}^{0}
$$

We can now distinguish two cases:

Case $1 h \leq v$ : Here, the two sets that hit at $\lambda_{2}=h$ have to be merged.

Case $2 h>v$ : In one of the sets, say $F_{i}$, for at least one edge $(k, l)$ with $k, l \in F_{i}$ we have $\tau_{k l}(v)= \pm v$ and if the slope remained unchanged, then $\left|\tau_{k l}\left(\lambda_{2}\right)\right|>\left|\lambda_{2}\right|$ for $\lambda_{2}>v$, thus the constraint would be violated. Then the capacity constraints of the associated maximum flow problem has to be updated and a new maximum flow identified. If for the new flow, all source edges are at capacity, only the trajectories for the $\tau_{k l}$ have to be altered. Otherwise, the set has to be split.

Therefore, we have defined the $\Delta$ of the previous theorem and identified the values of $\lambda_{2}$ at which the piecewise-linear solution path has breakpoints.

\subsubsection{Adapting the sets of fused variables}

Of course, we still have to specify how to exactly split a set for which the source edges are not at capacity into two smaller subsets. Assume that $F_{i}$ is the set that has to be split with associated maximum flow graph $\tilde{\mathcal{G}}_{i}$. Then define the set

$$
R_{i}=\left\{l \in F_{i}: r \text { connected to } l \text { by an augmenting path in } \tilde{\mathcal{G}}_{i}\right\}
$$

where the augmenting path is defined with respect to the maximal flow $f_{k l}$, i.e. for each node $l \in R_{i}$ there exists a path from the source $r$ to $l$ using only edges for which the flow is not at capacity. The complement of $R_{i}$ with respect to $F_{i}$ is defined as $S_{i}=F_{i} \backslash R_{i}$. Then we divide the set $F_{i}$ into the two subsets $R_{i}$ and $S_{i}$.

Now it remains to be shown that fusing or splitting sets as described above will yield sets of fused variables that satisfy the assumptions of Theorem 3. In particular, whenever we are at a breakpoint, i.e. have to fuse or split sets or both, we propose the following procedure for adapting the sets of fused variables:

1. If there are sets $F_{i}$ and $F_{j}$ for which $\exists k \in F_{i}$ and $l \in F_{j}$ with $(k, l) \in E$ and $\beta_{F_{i}}=\beta_{F_{j}}$, then fuse these sets into a new set $\tilde{F}_{i j}=F_{i} \cup F_{j}$ if $\left(\partial \beta_{F_{i}} / \partial \lambda_{2}\right)-\left(\partial \beta_{F_{j}} / \partial \lambda_{2}\right) \leq 0$ and $t_{k l}=1$.

2. If there is a set $F_{i}$ for which in the associated maximal flow graph not all edges coming from the source are at maximal capacity, then split $F_{i}$ in the two subsets $R_{i}$ and $S_{i}$ as described above.

3. Iterate steps 2 and 3 until nothing changes.

Using this procedure we can now show that adapting the sets in this way is correct. 


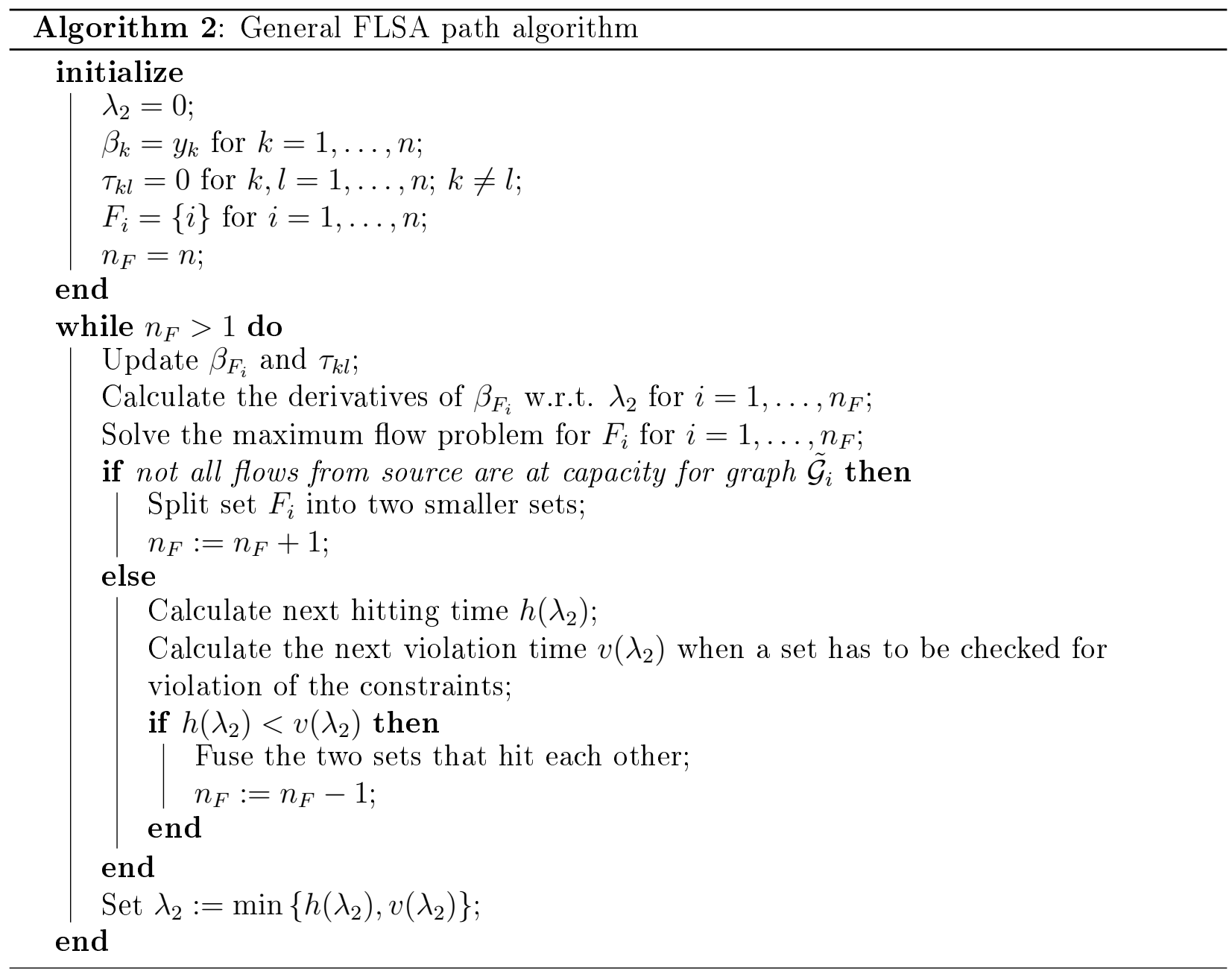

Proposition 1. Assume that we perform the fusion and split steps as described above. Then, the algorithm stops after a finite number of fusion and splitting steps and the resulting sets of variables are valid and satisfy the assumptions of Theorem 3.

Again, the proof can be found in the Online Supplement in Section 3. Putting all this together, we have shown that the solutions are piecewise linear and how to change the sets of fused variables at the breakpoints. So overall, using this algorithm we can calculate the entire solution path.

\subsection{Outline of the algorithm}

In the previous sections, we have seen how to derive the entire solution path of the general Fused Lasso Signal Approximator. Overall, the algorithm is very similar to the onedimensional FLSA outlined in Algorithm 1. The most important change is that, instead of only considering the fusion of sets, it is also necessary to track if it is necessary to break a set up into two smaller sets. Putting everything from the previous sections together, we get an outline of the algorithm for the general FLSA.

It should be noted that this is a basic outline of the algorithm and there is room for considerable efficiency gains when implementing it. Most importantly, similar to the onedimensional case from above, the hitting times $h_{i, j}$ only have to be updated if either the set 
$F_{i}$ or $F_{j}$ have changed since the last calculation. The same is true for the maximum flow problems. The flows only have to be updated if the underlying set has changed or a violation of a constraint was triggered. Therefore, in every iteration only a small number of sets is involved in the calculation and the computations can be done quickly. Especially for larger sets, the computationally most expensive step is solving the maximum flow problem. This gives us the possibility to derive an approximate version of the algorithm that is much faster as we will see in the simulations section below.

\subsection{Approximate algorithm}

The algorithm described above gives an exact solution to the problem. However, for large sets of fused variables, the calculation of a maximal flow is a bottleneck. In addition to this, if large sets of fused variables split, the resulting sets tend to be very unequal in size, often only splitting off a couple of nodes on the edges. Therefore, a lot of time is spent on cases that do not influence the solution very much. In order to speed up the algorithm, we propose to not check sets of fused variables for splitting up once they are larger than a certain size $K$. Then, for any set of size $K$ or larger, the maximum flow problem does not have to be solved, saving time on these especially computationally expensive sets. Also, as the values of $\tau_{k l}$ are only used to determine when a set has to be split, these also do not have to be updated any more for the large sets. As we will see in the simulations section, the tradeoff in accuracy for moderate values of $K$ is not very large but the algorithm speeds up considerably.

\subsection{Simulations}

In order to evaluate the performance of our exact and approximate algorithms above, we want to compare its speed and accuracy to the approximate FLSA algorithm for the 2-dimensional case presented in Friedman et al. [2007] as well as CVX (see Grant and Boyd [2008a,b]). As we also want to compare the accuracy of the approximate algorithms, we will use these techniques on simulated datasets, which we describe in more detail below.

The comparisons between the algorithms will be performed on datasets of various sizes ranging from $10 \times 10$ to $200 \times 200$. On each of these datasets, the solution will be computed for 50 equally spaced values of $\lambda_{2}$ between 0 and 0.5. For this speed comparison, there are two things to note.

First, as noted before, $C V X$ cannot use the solution of a similar $\lambda_{2}$ as a "warm start" to speed up computation. So, computing the solution for all 50 values of $\lambda_{2}$ takes roughly 50 times as long as computing the solution for just one value of $\lambda_{2}$. However, we chose to use it nonetheless as it is an easy to use general convex solver that can handle sparse matrices and is therefore equipped to handle large datasets.

Second, the algorithm that is presented in this paper not only calculates the solution at the 50 values of $\lambda_{2}$, but at all breakpoints of the piecewise-linear solution. The whole path is saved in a compact format and can be used to extract the solution at other values of $\lambda_{2}$ later much faster. For our dataset here, the solution path has at least as many breakpoints as there are datapoints, i.e. $n^{2}$ for an $n \times n$ grid. However, we still only let the other algorithm evaluate it for 50 values of $\lambda_{2}$ as we deemed it unrealistic that the solution for possibly thousands of $\lambda_{2}$ values is needed. 


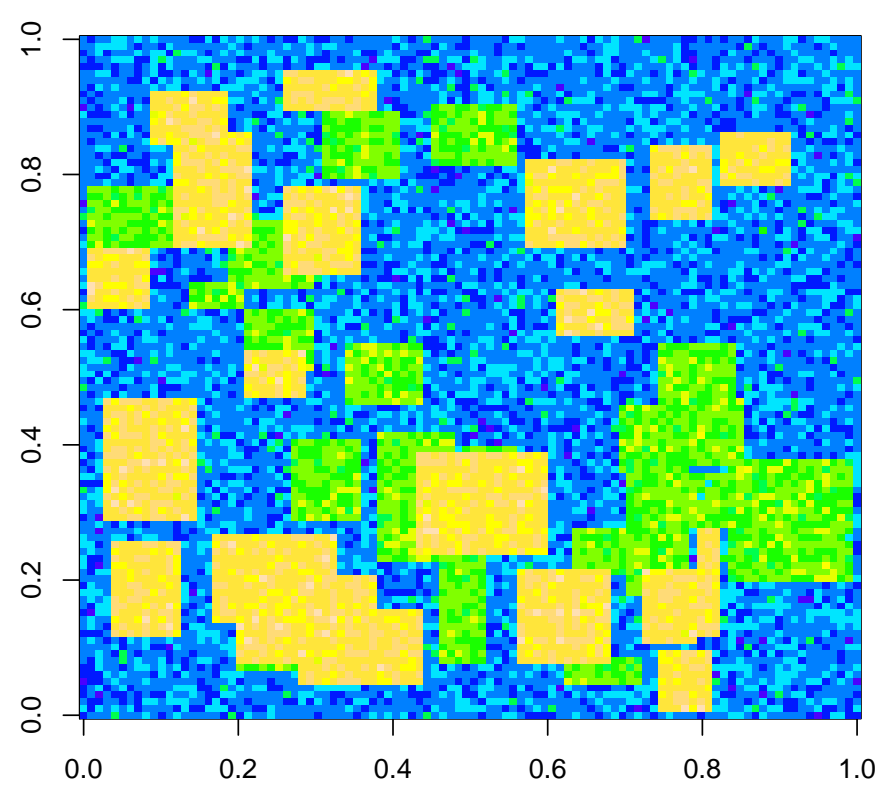

Figure 4: A sample image of the simulated 2-dimensional dataset

\subsubsection{The dataset}

In our comparisons, we want to use the 2-dimensional FLSA. Therefore, our data will consist of data $\mathbf{y}=\left\{y_{k l}\right\}$ with $k=1, \ldots, n_{1}$ and $l=1, \ldots, n_{2}$ with corresponding coefficients $\beta_{k l}$. The difference of coefficients will be penalized if they are neighbors on the 2-dimensional grid (horizontal or vertical), i.e. the loss function we want to minimize is

$$
L(\mathbf{y}, \boldsymbol{\beta})=\frac{1}{2} \sum_{k=1}^{n_{1}} \sum_{l=1}^{n_{2}}\left(y_{k l}-\beta_{k l}\right)^{2}+\sum_{k=1}^{n_{1}-1} \sum_{l=1}^{n_{2}}\left|\beta_{k l}-\beta_{k+1, l}\right|+\sum_{k=1}^{n_{1}} \sum_{l=1}^{n_{2}-1}\left|\beta_{k l}-\beta_{k, l+1}\right| .
$$

where we have already set $\lambda_{1}=0$. As shown above, we can get the solution for any $\lambda_{1}$ by soft-thresholding the solution for $\lambda_{1}=0$. In our simulated dataset, we set $n_{1}=n_{2}=n$ for various values of $n$. The value of $y_{k l}$ is being generated as follows:

1. Set $y_{k l}=0$ for all $k, l=1, \ldots, n$.

2. For some rectangles of random size, change the value of $y$ to either 1 or 2 , such that roughly $20 \%$ have value 1 and $20 \%$ have value 2 .

3. To every point $y_{k l}$ add standard normal noise with standard deviation of 0.2 .

A sample image of what the simulated dataset looks like can be seen in Figure 4.

\subsubsection{Results}

First, we compare the computation time of the three methods. The results can be seen in Table 2. The path algorithm as well as the component-wise algorithm are both much faster 


\begin{tabular}{|c|c|c|c|c|c|}
\hline \multicolumn{2}{|c|}{ Image size } & $10 \times 10$ & $50 \times 50$ & $100 \times 100$ & $200 \times 200$ \\
\hline \multicolumn{2}{|c|}{ CVX } & 36 & 140 & 1000 & 6800 \\
\hline \multicolumn{2}{|c|}{ Component-wise Alg. } & 0.062 & 0.61 & 2.2 & 7.7 \\
\hline \multirow{11}{*}{ Path Alg. } & $K=1$ & 0.0031 & 0.17 & 1.1 & 10 \\
\hline & $K=2$ & 0.0032 & 0.18 & 1.1 & 10 \\
\hline & $K=5$ & 0.0040 & 0.20 & 1.3 & 10 \\
\hline & $K=10$ & 0.0046 & 0.23 & 1.4 & 11 \\
\hline & $K=50$ & 0.0077 & 0.37 & 2.0 & 14 \\
\hline & $K=100$ & - & 0.51 & 3.0 & 18 \\
\hline & $K=500$ & - & 1.3 & 11 & 62 \\
\hline & $K=1000$ & - & 1.4 & 21 & 120 \\
\hline & $K=2000$ & - & 1.4 & 22 & 290 \\
\hline & $K=5000$ & - & - & 23 & 400 \\
\hline & exact & 0.0079 & 1.4 & 24 & - \\
\hline
\end{tabular}

Table 2: Speed comparison for the 2-dimensional FLSA in seconds. The solution is evaluated for 50 values of $\lambda_{2}$ between 0 and 0.5 . The results are averaged over 10 runs for the $10 \times 10$ and $50 \times 50$ dataset and 4 simulation runs for the rest.

than the general convex solver CVX. When comparing the path algorithm to the componentwise algorithm, we see that they have roughly the same speed for low values of $K$, except for the small $10 \times 10$ dataset.

With respect to accuracy, we measure both the sup-norm error as well as the root mean squared deviation (RMSD). These measures are being calculated for each value of $\lambda_{2}$ and the largest values, averaged over several simulation runs are being displayed in Tables 3 and 4. CVX returns the exact solution in all cases, although at the cost of a rather slow speed. The component-wise algorithm on the other hand is quite fast, however only yields an approximate solution, although with a rather small error rate in terms of RMSD. With varying $K$, the path algorithm is in between the other two methods. However, for values of $K$ around $500-1000$, the path algorithm is very accurate but still a lot faster than CVX. For most practical application, this time-accuracy tradeoff may be worthwhile.

\section{Conclusion}

In this article we develop a path algorithm for the Fused Lasso Signal Approximator in its onedimensional and general form. We compared the speed and accuracy of the FLSA algorithm to other available methods and conclude that our method has advantages in terms of speed and the amount of information gathered and stored. Especially compared to standard convex solvers, our path algorithm is much faster for the FLSA. It is also very easy and quick to extract results for additional penalty parameter values. We also extend this work to the case of the general Fused Lasso where we restrict ourselves to predictor matrices with $\operatorname{rank}(X)=p$ where $X \in \mathbb{R}^{n \times p}$ (see the Online Supplement).

Apart from the work presented here, there are several ways how we plan to expand on it in the future. It is possible to expand the Fused Lasso by allowing each summand in the penalty terms to have separate weights, i.e. a loss function of the form 


\begin{tabular}{|c|c|c|c|c|c|}
\hline \multicolumn{2}{|c|}{ Image size } & $10 \times 10$ & $50 \times 50$ & $100 \times 100$ & $200 \times 200$ \\
\hline \multicolumn{2}{|c|}{ CVX } & 0 & 0 & 0 & 0 \\
\hline \multicolumn{2}{|c|}{ Component-wise Alg. } & 0.30 & 0.80 & 0.73 & 1.0 \\
\hline \multirow{11}{*}{ Path Alg. } & $K=1$ & 0.31 & 0.81 & 0.73 & 1.0 \\
\hline & $K=2$ & 0.26 & 0.75 & 0.73 & 1.0 \\
\hline & $K=5$ & 0.22 & 0.74 & 0.73 & 0.90 \\
\hline & $K=10$ & 0.15 & 0.68 & 0.73 & 0.90 \\
\hline & $K=50$ & 0 & 0.44 & 0.22 & 0.52 \\
\hline & $K=100$ & 0 & 0.35 & 0.18 & 0.43 \\
\hline & $K=500$ & 0 & 0.025 & 0.047 & 0.14 \\
\hline & $K=1000$ & 0 & 0 & 0.013 & 0.12 \\
\hline & $K=2000$ & 0 & 0 & 0.00017 & 0.050 \\
\hline & $K=5000$ & 0 & 0 & 0.00017 & 0.021 \\
\hline & exact & 0 & 0 & 0 & - \\
\hline
\end{tabular}

Table 3: Absolute error accuracy comparison for the 2-dimensional FLSA. The accuracy of the approximate version of the path algorithm and the component-wise algorithm are compared to the exact solution using the supremum norm. The largest error of the 50 values of $\lambda_{2}$ is reported. The results are averaged over 10 runs for the $10 \times 10$ and $50 \times 50$ dataset and 4 simulation runs for the rest.

\begin{tabular}{|c|c|c|c|c|c|}
\hline \multicolumn{2}{|c|}{ Image size } & $10 \times 10$ & $50 \times 50$ & $100 \times 100$ & $200 \times 200$ \\
\hline \multicolumn{2}{|c|}{ CCVX } & 0 & 0 & 0 & 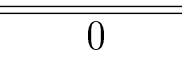 \\
\hline \multicolumn{2}{|c|}{ Component-wise Alg. } & 0.056 & 0.066 & 0.041 & 0.030 \\
\hline \multirow{11}{*}{ Path Alg. } & $K=1$ & 0.059 & 0.067 & 0.045 & 0.031 \\
\hline & $K=2$ & 0.056 & 0.066 & 0.044 & 0.030 \\
\hline & $K=5$ & 0.041 & 0.059 & 0.042 & 0.029 \\
\hline & $K=10$ & 0.029 & 0.053 & 0.039 & 0.027 \\
\hline & $K=50$ & $<10^{-5}$ & 0.035 & 0.027 & 0.020 \\
\hline & $K=100$ & 0 & 0.022 & 0.022 & 0.015 \\
\hline & $K=500$ & 0 & 0.0050 & 0.0059 & 0.0078 \\
\hline & $K=1000$ & 0 & $<10^{-5}$ & 0.00026 & 0.0053 \\
\hline & $K=2000$ & 0 & $<10^{-5}$ & $<10^{-5}$ & 0.0028 \\
\hline & $K=5000$ & 0 & 0 & $<10^{-5}$ & 0.0019 \\
\hline & exact & 0 & 0 & 0 & - \\
\hline
\end{tabular}

Table 4: Root mean square deviation (RMSD) accuracy comparison for the 2-dimensional FLSA. The accuracy of the approximate version of the path algorithm and the componentwise algorithm are compared to the exact solution using the RMSD. The largest error of the 50 values of $\lambda_{2}$ is reported. The results are averaged over 10 runs for the $10 \times 10$ and $50 \times 50$ dataset and 4 simulation runs for the rest. 


$$
L_{\lambda_{1}, \lambda_{2}}(y, X, \beta)=\frac{1}{2}(y-X \beta)^{T}(y-X \beta)+\lambda_{1} \sum_{k=1}^{p} w_{k}\left|\beta_{k}\right|+\lambda_{2} \sum_{(k, l) \in E, k<l} w_{k l}\left|\beta_{k}-\beta_{l}\right| .
$$

This more complicated model can be solved by a generalization of the algorithms presented in this article. Also, the current algorithm for the FLSA is not optimized form graphs with large number of edges in them. We plan to develop a version that takes cliques into account to achieve further efficiency gains.

We hope that our algorithms will be used to analyze data and as a building block for other new models. In order to facilitate this we will be publishing implementations of the algorithms in the form of an R package on CRAN and the authors website.

\section{References}

Dimitri P. Bertsekas. Nonlinear Programming. Athena Scientific, 1999.

Thomas H. Cormen, Charles E. Leierson, Ronald L. Rivest, and Clifford Stein. Introduction to Algorithms. MIT Press and McGraw-Hill, second edition edition, 2001.

Bradley Efron, Trevor Hastie, Ian Johnstone, and Robert Tibshirani. Least angle regression. Annals of Statistics, 32(2):407-499, 2004.

J. Friedman, T. Hastie, H. Hoefling, and R. Tibshirani. Pathwise coordinate optimization. Annals of Applied Statistics, 2007.

M. Grant and S. Boyd. Cvx: Matlab software for disciplined convex programming (web page and software). http://stanford.edu/ boyd/cvx, June 2008a.

M. Grant and S. Boyd. Graph implementations for nonsmooth convex programs. Springer, 2008b. Recent Advances in Learning and Control (tribute to M. Vidyasagar).

Trevor Hastie, Saharon Rosset, Robert Tibshirani, and Ji Zhu. The entire regularization path for the support vector machine. The Journal of Machine Learning Research, 5:1391-1415, 2004 .

Mee-Young Park and Trevor Hastie. $l_{1}$-regularization path algorithm for generalized linear models. JRSSB, 69:659-677, 2007.

Saharon Rosset and Ji Zhu. Piecewise linear regularized solution paths. Annals of Statistics, 35(3):1012-1030, 2007.

Robert Tibshirani. Regression shrinkage and selection via the lasso. Journal of the Royal Statistical Society Series B, 58:267-288, 1996.

Robert Tibshirani, Michael Saunders, Ji Zhu, and Saharon Rosset. Sparsity and smoothness via the fused lasso. JRSSB, 67:91-108, 2005. 


\title{
Supplement to "A path algorithm for the Fused Lasso Signal Approximator"
}

\author{
Holger Hoefling* \\ Stanford University
}

October 3, 2009

\section{Introduction}

In this online supplement to the article "A path algorithm for the Fused Lasso", we will give proofs and further results that would have gone beyond the possible size and scope of the paper. In Section 2 we will discuss the computational and memory complexity of the algorithm. In addition to this we will give an alternative proof to Theorem 2 of the article in which we show that for increasing $\lambda_{2}$, fused sets of variables do not become unfused again. In Section 3 we will give the proofs to Theorem 3 and Proposition 1 that complete our results for the general Fused Lasso Signal Approximator. Finally, in Section 4, we will extend the path algorithm of the FLSA to the case of the general Fused Lasso where for the matrix of predictors $\mathbf{X} \in \mathbb{R}^{n \times p}$ we have $\operatorname{rank}(\mathbf{X})=p$ and we also present the associated proofs.

\section{One dimensional FLSA}

\subsection{Computational complexity}

In Section 2 of the article, we have presented the algorithm that solves the one-dimensional Fused Lasso Signal Approximator. We stated that the computational complexity of the algorithm is $n \log (n)$. In order to calculate the complexity of the algorithm, first note that the initialization step takes $2 n+2$ operations. However, most of the computations are performed for calculating the next hitting time $h\left(\lambda_{2}\right)$. When $\lambda_{2}=0$, the derivative $\partial \beta_{F_{i}} / \partial \lambda_{2}$ has to be computed for all $n$ groups and the smallest hitting time identified. This takes on the order $O(n)$ operations. However, in subsequent steps, $h_{i, i+1}$ remain the same except if either $F_{i}$ or $F_{i+1}$ was fused with its neighbor in the last iterations. This way, in each iteration only 2 values of $h_{i, i+1}$ have to be updated. Finding the smallest value of $h_{i, i+1}$ requires operations on the order $O(\log (n))$ if an efficient data structure is used to save the $h_{i, i+1}$ (e.g. a binary tree). The same is true for the updates of the $\beta_{F_{i}}$. Only the $\beta_{F_{i}}$ of the groups that have just been fused have to be updated. For the other groups $\partial \beta_{F_{i}} / \partial \lambda_{2}$ stays the same, so we can interpolate $\beta_{F_{i}}\left(\lambda_{2}\right)$ when we need it based on $\lambda_{2}^{0}$ when this group was created as well as $\beta_{F_{i}}\left(\lambda_{2}^{0}\right)$ and its derivative. So this also only involves a constant number of operations per

*Email: hhoeflin@gmail.com 


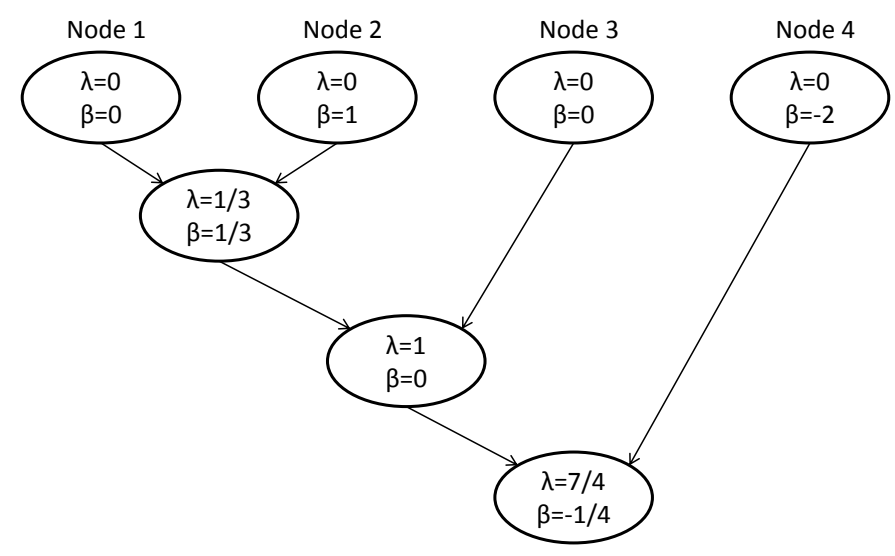

Figure 1: Example of a tree for storing the solution path of the one-dimensional FLSA.

iteration. As with every iteration, the number of sets $n_{F}$ decreases by one, there are at most $n-1$ iterations. Therefore, the entire solution path can be obtained with a computational complexity on the order $O(n \log (n))$.

\subsection{Storing the solution path efficiently}

One possibility to store and retrieve the optimal coefficients for a value of $\lambda_{2}$ would be to store the whole coefficient vector $\boldsymbol{\beta}$ for every value of $\lambda_{2}$ for which 2 sets are fused and interpolate linearly in between. However, then it would be necessary to store $n^{2}$ entries, which would be impossible for large values of $n$. However, when done efficiently, it is possible to store the entire solution with a memory requirement that only grows linearly in $n$. For this, the result is stored in the form of a binary tree. Every node contains a value for $\lambda_{2}$ at which this node (corresponding to a set of fused variables) becomes active and the correct value of $\beta_{F_{i}}$ for this same value $\lambda_{2}$.

We start by creating $n$ unconnected nodes corresponding to the $n$ coefficients for $\lambda_{2}=0$ and $\beta_{k}=y_{k}$. Then when two sets of coefficients fuse, add a new node that is the parent of the two nodes corresponding to the 2 groups that were just fused. In the new node, store the value of $\lambda_{2}$ as well as the updated value of the coefficient $\beta_{F_{i}}$. At the end of the algorithm, we will have one binary tree (for a small example see Figure 1). In order to retrieve one value of the solution at $\tilde{\lambda}_{2}$ for coefficient $\beta_{k}$, first start at the leaf node of coefficient $\beta_{k}$. Climb up the tree until $\lambda_{2}$ of the next parent node is larger than $\tilde{\lambda}_{2}$. The correct solution is then a linear interpolation between the $\beta_{F_{i}}$ values stored in the current node and the parent node. Using this method, the complexity for looking the whole vector of solutions $\beta$ for a particular value of $\lambda_{2}$ is then also $O(n \log n)$. Therefore, running the path algorithm and getting a solution vector has a total complexity of $O(n \log n)$. We will also be able to see this in the next section when we compare the speed of this algorithm to other existing methods for this problem.

\subsection{Alternative proof of Theorem 2}

An important prerequisite for the algorithm is the result of Theorem 2 in the article. A proof has already been given in Friedman et al. [2007] and here we want to give an alternative 
version. It is based on the results of the Genreal Fused Lasso Signal Approximator, so read Section 3 of the article before this proof.

Theorem 2 For the one-dimensional FLSA assume that for some $\lambda_{2}^{0}>0$ and $\lambda_{1}=0$, we have that $\beta_{k}\left(\lambda_{2}^{0}\right)=\beta_{k+1}\left(\lambda_{2}^{0}\right)$ for some $1 \leq k \leq N-1$. Then, for any $\lambda_{2}>\lambda_{2}^{0}$ we have that $\beta_{k}\left(\lambda_{2}\right)=\beta_{k+1}\left(\lambda_{2}\right)$.

Proof. First note that in Section 3.2.2, we described the maximum flow problem that we have to solve and under which conditions it is necessary to break up the set of fused variables. In the case of the one-dimensional FLSA, the maximum flow graph is especially simple. Assume that an arbitrary set of fused variables $|F|$ is $\left\{k_{0}, k_{0}+1, \ldots, k_{0}+|F|-1\right\}$, which has the edges $\left\{\left(k_{0}, k_{0}+1\right), \ldots,\left(k_{0}+|F|-2, k_{0}+|F|-1\right)\right\}$. For the capacities on these edges, it is sufficient to note that they are always $\geq 1$. Now it only remains to specify which edges are connected to the source node $r$ and the sink node $s$ and which capacities they have. Essentially, there are 4 possible situations:

Case $1 \beta_{k_{0}-1}, \beta_{k_{0}+|F|}>\beta_{F}$ : In this case we have that $\frac{\partial \beta_{F}}{\partial \lambda_{2}}=\frac{2}{|F|}$. Then we have that $k_{0}$ and $k_{0}+|F|-1$ are connected to the source $r$ and all other nodes are connected to the sink $s$. For the capacities from the source, we have $c_{r l}=1-\frac{2}{|F|} \leq 1$ and for the capacities to the sink it holds that $c_{k s}=\frac{2}{|F|} \leq 1$. As the capacities on the inner nodes is at least 1 as stated above, it is easy to see that for the maximum flow we always have $f_{r l}=c_{r l}$ for $l=k_{0}, k_{0}+|F|-1$ and therefore the group will never be split.

Case $2 \beta_{k_{0}+1}>\beta_{F}>\beta_{k_{0}+|F|}$ : Here, we have that $\frac{\partial \beta_{F}}{\partial \lambda_{2}}=0$ and node $k_{0}$ is connected to the source $r$ whereas $k_{0}+|F|-1$ is connected to the sink $s$. All other nodes are not connected to either the source or the sink. For the capacities in this case we have $c_{r k_{0}}=c_{k_{0}+|F|-1, s}=1$ and therefore the maximum flow is always $f_{r k_{0}}=f_{k_{0}, k_{0}+1}=\cdots=$ $f_{k_{0}+|F|-2, k_{0}+|F|-1}=f_{k_{0}+|F|-1, s}=1$ and therefore, the group will never be split.

Case $3 \beta_{k_{0}+1}<\beta_{F}<\beta_{k_{0}+|F|}$ : Similar to case 2 .

Case $4 \beta_{k_{0}-1}, \beta_{k_{0}+|F|}<\beta_{F}$ : Similar to case 1 .

In these cases we assumed that $k_{0} \neq 1$ and $k_{0}+|F|-1 \neq N$, i.e. that the set of fused variables is not at the boundary of the graph. However, it is easy to see that in this case the set will also not break apart for increasing $\lambda_{2}$. Therefore, the proposition holds.

\section{The general Fused Lasso Signal Approximator}

In this section we want to give the proofs of Theorem 3 and Proposition 1 of the article. First, we will give the proof of Theorem 3, then the one of Proposition 1.

\subsection{Proof of Theorem 3}

Proof. In order to show that this is indeed the solution, we have to show that for any $\lambda_{2} \in\left[\lambda_{2}^{0}, \lambda_{2}^{0}+\Delta\right]$, the subgradient Equations (5) hold. We will do this for every group $F_{i}$ 
separately. We know that

$$
\frac{\partial \beta_{F_{i}}}{\partial \lambda_{2}}=-\frac{1}{\left|F_{i}\right|} \sum_{j \neq i} \sum_{(k, l) \in E ; k \in F_{i} ; l \in F_{j}} t_{k l} .
$$

For the maximum flow graph, we know that all nodes except the source and the sink have a net flow of 0 . Furthermore, we also know that the sum of all capacities coming from the source is the same as the sum of all capacities going to the sink as

$$
\sum_{(r, l) \in \tilde{E}_{i}} c_{r l}-\sum_{(k, s) \in \tilde{E}_{i}} c_{k s}=\sum_{k \in F_{i}} p_{k}=0
$$

where we use that for any node that is not connected to either the source or the sink, $p_{k}=0$. From this we can see that if all flows coming from the source are at maximum capacity, so are all flows going to the sink. Therefore, for a maximum flow with all flows from the source being at maximum capacity, we have

$$
\sum_{l:(k, l) \in E ; l \in F_{i}} f_{k l}= \begin{cases}-f_{k r}=c_{r k}=p_{k} & \text { if }(r, k) \in \tilde{E}_{i} \\ -f_{k s}=-c_{k s}=p_{k} & \text { if }(k, s) \in \tilde{E}_{i} \\ 0=p_{k} & \text { otherwise }\end{cases}
$$

so that overall we get

$$
\begin{array}{r}
\beta_{k}\left(\lambda_{2}\right)-y_{k}+\lambda_{2} \sum_{l:(k, l) \in E} t_{k l}\left(\lambda_{2}\right) \\
=\beta_{k}\left(\lambda_{2}^{0}\right)-y_{k}+\lambda_{2} \sum_{l:(k, l) \in E} t_{k l}\left(\lambda_{2}^{0}\right)+\frac{\partial \beta_{F_{i}}}{\partial \lambda_{2}}\left(\lambda_{2}-\lambda_{2}^{0}\right) \\
+\sum_{l \in F_{i}:(k, l) \in E} f_{k l}\left(\lambda_{2}-\lambda_{2}^{0}\right)+\sum_{l \notin F_{i}:(k, l) \in E} t_{k l}\left(\lambda_{2}^{0}\right)\left(\lambda_{2}-\lambda_{2}^{0}\right) \\
=\left(\lambda_{2}-\lambda_{2}^{0}\right)\left[\frac{\partial \beta_{F_{i}}}{\partial \lambda_{2}}+\sum_{l \in F_{i}:(k, l) \in E} f_{k l}+\sum_{l \notin F_{i}:(k, l) \in E} t_{k l}\left(\lambda_{2}^{0}\right)\right] \\
=\left(\lambda_{2}-\lambda_{2}^{0}\right)\left[\sum_{l \in F_{i}:(k, l) \in E} f_{k l}-p_{k}\right]=0
\end{array}
$$

where we use the definition of $p_{k}\left(\lambda_{2}^{0}\right)=-\sum_{l \notin F_{i}:(k, l) \in E} t_{k l}\left(\lambda_{2}^{0}\right)-\frac{\partial \beta_{F_{i}}}{\partial \lambda_{2}}$ in the second to last equality. As this is true for every $k \in F_{i}$ and group $F_{i}$, the solution as proposed in the theorem solves the subgradient equations and therefore minimizes the loss function.

\subsection{Proof of Proposition 1}

Proof. First, we want to show that after the fusion and splitting steps finish, we have a valid set of fused variables. For this, we have to show that for sets $F_{i}$ and $F_{j}$ it holds that $\beta_{F_{i}}\left(\lambda_{2}\right) \neq \beta_{F_{j}}\left(\lambda_{2}\right)$ for $\lambda_{2} \in\left(\lambda_{2}^{0}, \lambda_{2}^{0}+\varepsilon\right)$ for some $\varepsilon>0$. However, this is certainly true. If $\beta_{F_{i}}\left(\lambda_{2}^{0}\right) \neq \beta_{F_{j}}\left(\lambda_{2}^{0}\right)$, then this holds also for some interval $\lambda_{2} \in\left(\lambda_{2}^{0}, \lambda_{2}^{0}+\varepsilon\right)$ as $\beta_{k}\left(\lambda_{2}\right)$ is 
continuous in $\lambda_{2}$. If we have that $\beta_{F_{i}}\left(\lambda_{2}^{0}\right)=\beta_{F_{j}}\left(\lambda_{2}^{0}\right)$, then we can assume that $\frac{\partial \beta_{F_{i}}}{\partial \lambda_{2}}\left(\lambda_{2}^{0}\right)>$ $\frac{\partial \beta_{F_{j}}}{\partial \lambda_{2}}\left(\lambda_{2}^{0}\right)$ and $t_{k l}=1$ for $k \in F_{i}, l \in F_{j} ;(k, l) \in E$, as otherwise $F_{i}$ and $F_{j}$ would have been fused. However, this means that $\beta_{F_{i}}\left(\lambda_{2}\right)>\beta_{F_{j}}\left(\lambda_{2}\right)$ for $\lambda_{2} \in\left(\lambda_{2}^{0}, \lambda_{2}^{0}+\varepsilon\right)$ and that $t_{k l}\left(\lambda_{2}\right)=\operatorname{sign}\left(\beta_{F_{i}}\left(\lambda_{2}\right)-\beta_{F_{j}}\left(\lambda_{2}\right)\right)$, so the grouping is valid.

Second, for all of the sets at the end of the splitting steps, the maximal flow condition of Theorem 3 trivially holds as any set for which it doesn't hold, would be split up into two smaller sets (and it always holds for sets of size 1).

Therefore, it only remains to show that the fusion and splitting steps converge after a finite number of iterations. For this, we show that if a set $F_{i}$ was split into subgroups $R_{i}$ and $S_{i}$ at penalty parameter $\lambda_{2}^{0}$, then $R_{i}$ and $S_{i}$ will not be merged at $\lambda_{2}^{0}$ in a subsequent iteration. From this, we will conclude that there is only a finite number of possible iterations, as there is only a finite number of possible sets and we cannot have infinite cycles of fusions and splits for the sets. Therefore, the algorithm converges after a finite number of steps.

So, as $F_{i}$ was split into $R_{i}$ and $S_{i}$, we know that $R_{i} \neq \emptyset$ as well as $S_{i} \neq \emptyset$. For set $R_{i}$ consider the capacities of edges the source into $R_{i}$ minus the capacities of edges going from $R_{i}$ either to $S_{i}$ or directly to the sink. As we split group $F_{i}$, we know that the capacities going into $R_{i}$ are larger than the capacities going out and therefore,

$$
\sum_{(r, l) \in \tilde{E}_{i} ; l \in R_{i}} c_{r l}>\sum_{(k, l) \in \tilde{E}_{i} ; k \in R_{i}, l \in S_{i}} c_{k l}+\sum_{(k, s) \in \tilde{E}_{i} ; k \in R_{i}} c_{k s} .
$$

For all edges $(k, l) \in \tilde{E}_{i}$ with $k \in R_{i}$ and $l \in S_{i}$ we also know that $1=c_{k l}=f_{k l}=t_{k l}$. Here $f_{k l}=c_{k l}$ follows as otherwise $l$ would be connected to the source in the residual graph and therefore $l \in R_{i}$, which is not the case as $l \in S_{i}$. Also, it follows that $c_{k l}=1$, as $c_{k l}=\infty$ is the only other option which cannot hold as the maximum flow is finite. Furthermore $t_{k l}=1$ as $c_{k l}=1$. Then

$$
\sum_{(r, l) \in \tilde{E}_{i} ; l \in R_{i}} c_{r l}-\sum_{(k, s) \in \tilde{E}_{i} ; k \in R_{i}} c_{k s}-\sum_{(k, l) \in \tilde{E}_{i} ; k \in R_{i}, l \in S_{i}} t_{k l}>0 .
$$

Using that $c_{r l}=p_{l}$ for $(r, l) \in \tilde{E}_{i}$ and $c_{k s}=-p_{k}$ for $(k, s) \in \tilde{E}_{i}$, we get

$$
\sum_{k \in R_{i}} p_{k}-\sum_{(k, l) \in \tilde{E}_{i} ; k \in R_{i}, l \in S_{i}} t_{k l}>0 .
$$

Let $p_{k}^{R_{i}}$ be the push on node $k$ in the graph associated with group $R_{i}$ and $p_{l}^{S_{i}}$ the push on node $l$ associated with $S_{i}$ after the split. From equation (8), we know that

$$
\sum_{k \in R_{i}} p_{k}^{R_{i}}=0
$$

We can also infer from the definition of the push on $F_{i}$ and $R_{i}$ that

$$
\sum_{k \in R_{i}} p_{k}^{R_{i}}+\left|R_{i}\right| \frac{\partial \beta_{R_{i}}}{\partial \lambda_{2}}=\sum_{k \in R_{i}} p_{k}-\sum_{k \in R_{i}, l \in S_{i},(k, l) \in E} t_{k l}+\left|R_{i}\right| \frac{\partial \beta_{F_{i}}}{\partial \lambda_{2}}
$$


and therefore using equation (1) and $\sum_{k \in R_{i}} p_{k}^{R_{i}}=0$ that

$$
\frac{\partial \beta_{R_{i}}}{\partial \lambda_{2}}>\frac{\partial \beta_{F_{i}}}{\partial \lambda_{2}}
$$

Now, again using equation (8), we get

$$
\frac{\partial \beta_{F_{i}}}{\partial \lambda_{2}}=\frac{\left|R_{i}\right|}{\left|R_{i}\right|+\left|S_{i}\right|} \frac{\partial \beta_{R_{i}}}{\partial \lambda_{2}}+\frac{\left|S_{i}\right|}{\left|R_{i}\right|+\left|S_{i}\right|} \frac{\partial \beta_{S_{i}}}{\partial \lambda_{2}}
$$

and thus

$$
\frac{\partial \beta_{R_{i}}}{\partial \lambda_{2}}>\frac{\partial \beta_{F_{i}}}{\partial \lambda_{2}}>\frac{\partial \beta_{S_{i}}}{\partial \lambda_{2}}
$$

As also $t_{k l}=1$ for $k \in R_{i}$ and $l \in S_{i}$, we can see that the groups that have just been split at $\lambda_{2}^{0}$ cannot be merged again at the same penalty parameter $\lambda_{2}^{0}$.

In turn, this also means that any two sets that have just been fused, cannot be immediately split up again into the same original sets at $\lambda_{2}^{0}$ as this would lead us back to the starting position, which would force us to fuse the two sets again. However, we have just shown that this cannot happen and therefore we also see that any two sets that are being fused, are not being split up again immediately.

\section{Path algorithm for the General Fused Lasso}

In this section we want to expand the result of the article from a path algorithm of the general FLSA to a path algorithm for the general Fused Lasso with a predictor matrix $\mathbf{X} \in \mathbb{R}^{n \times p}$ where $\operatorname{rank}(\mathbf{X})=p$. The loss function we want to minimize in this case is

$$
L_{\lambda_{1}, \lambda_{2}}(\mathbf{y}, \mathbf{X}, \boldsymbol{\beta})=\frac{1}{2}(\mathbf{y}-\mathbf{X} \boldsymbol{\beta})^{T}(\mathbf{y}-\mathbf{X} \boldsymbol{\beta})+\lambda_{1} \sum_{k=1}^{p}\left|\beta_{k}\right|+\lambda_{2} \sum_{(k, l) \in E, k<l}\left|\beta_{k}-\beta_{l}\right|
$$

where $\mathbf{X} \in \mathbb{R}^{n \times p}$ and $\mathcal{G}=(V, E)$ a graph that defines the penalty structure on the difference of coefficients $\beta_{k}-\beta_{l}$ for $(k, l) \in E$, the set of undirected edges. One important difference to the $\mathbf{X}=\mathbf{I}$ case is that here, we cannot get the solution for all values of $\lambda_{1}$ by soft-thresholding the solutions for $\lambda_{1}=0$ as we did for the FLSA. Therefore, we cannot calculate one path from which it is easy to get all solutions for any combinations of $\left(\lambda_{1}, \lambda_{2}\right)$. Instead, we will restrict ourselves to calculate the whole solution path for $\lambda_{2}$ for a fixed value $\lambda_{1}$. Therefore, we have to incorporate the additional penalty $\lambda_{1} \sum_{k=1}^{p}\left|\beta_{k}\right|$ into the algorithm. This will be done in a fashion similar to the LARS algorithm (see Efron et al. [2004]) by having active and inactive sets of fused variables.

The reason that we restrict the development of the algorithm to the case of $\operatorname{rank}(X)=p$ is that if $\operatorname{rank}(X)<p$, then the resulting solution path w.r.t. $\lambda_{2}$ may have discontinuities. Here is a very small example where this happens. Assume that $n=1, p=2$ with $y_{1}=3$ as well as $x_{11}=1$ and $x_{12}=0.5$. For $\lambda_{1}=1$, the solution path for $\lambda_{2}$ can be seen in Figure 2 . As we can see, for $\lambda_{2}=1 / 3$, the solution path has a jump.

In this section, we will first enhance the definition of sets of fused variables to incorporate that these sets can now also be active or inactive. Next, we will show that the solution path is again piecewise linear and how to calculate its slope and the necessary changes at the 


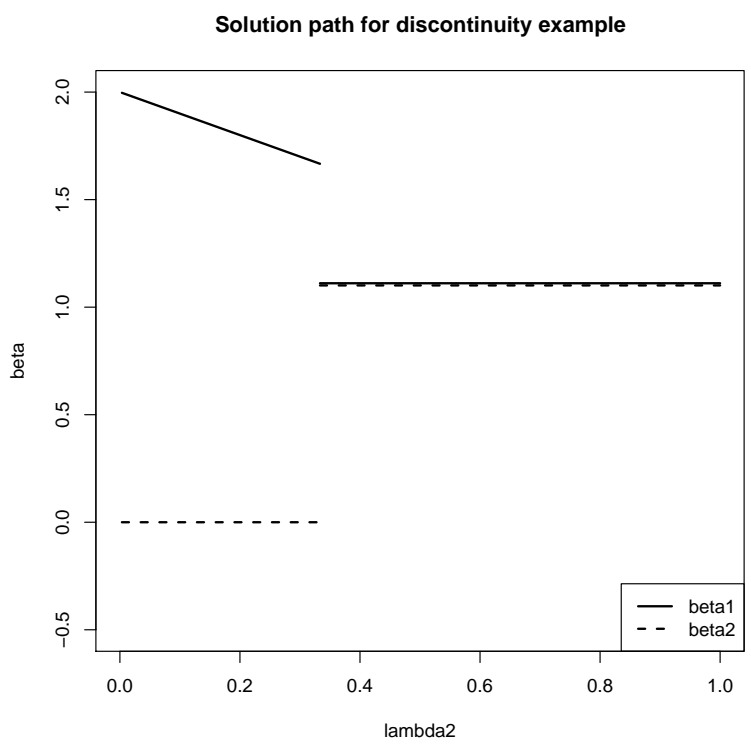

Figure 2: Example of a discontinuous solution path if $\operatorname{rank}(X)<p$.

breakpoints. We will end this section by giving the complete algorithm that calculates the solution path for $\lambda_{2}$ where $\lambda_{1}$ is assumed to be fixed.

\subsection{Sets of fused variables and active sets}

In the case of the general Fused Lasso, it is not possible to assume that $\lambda_{1}=0$ and therefore we have to account for the additional penalty $\lambda_{1} \sum_{k=1}^{p}\left|\beta_{k}\right|$ in the loss function. Therefore, in addition to sets of fused variables as in the case of the FLSA algorithm, it is also necessary to keep track if these sets $F_{i}$ are currently "active", that is have an associated coefficient $\beta_{F_{i}} \neq 0$, or inactive when $\beta_{F_{i}}=0$. In order to account for this, we have to adapt the definition of sets of variables. However, before we do this we should have a look at the $k$-th subgradient equation for the general loss function first:

$$
\frac{\partial L_{\lambda_{1}, \lambda_{2}}}{\partial \beta_{k}}=-\left(X^{T} y\right)_{k}+\left(X^{T} X \beta\right)_{k}+\lambda_{1} s_{k}+\lambda_{2} \sum_{l:(k, l) \in E} t_{k l}
$$

where $s_{k}=\operatorname{sign}\left(\beta_{k}\right)$ for $\beta_{k} \neq 0$ and $s_{k} \in[-1,1]$ otherwise. Also, as before $t_{k l}=\operatorname{sign}\left(\beta_{k}-\beta_{l}\right)$ for $\beta_{k} \neq \beta_{l}$ and $t_{k l} \in[-1,1]$ for $\beta_{k}=\beta_{l}$. Variables are considered fused if $\beta_{k}=\beta_{l}$ and unfused if $\beta_{k} \neq \beta_{l}$. They are active if $\beta_{k} \neq 0$, from which we see that in a set of fused variables, always all variables are either active or not. Thus, when "activating" coefficients, we always have to activate a whole set at once. In order to do this, we also have to keep track of sets of variables that are inactive by using $s_{k}$ as a substitute for $\beta_{k}$. More precisely:

Definition 3 Let $p_{F}\left(\lambda_{2}\right)$ be the number of sets of fused variables for penalty parameter $\lambda_{2}$ (keeping $\lambda_{1}$ fixed). Then for the sets $F_{i}, i=1, \ldots, p_{F}\left(\lambda_{2}\right)$ to be valid, the following conditions have to hold:

1. $\cup_{i=1}^{n_{F}\left(\lambda_{2}\right)} F_{i}=\{1, \ldots, n\}$ 
2. $F_{i} \cap F_{j}=\emptyset$ for $i \neq j$

3. If $k, l \in F_{i}$ then $\beta_{k}\left(\lambda_{2}\right)=\beta_{l}\left(\lambda_{2}\right)$ as well as $s_{k}\left(\lambda_{2}\right)=s_{l}\left(\lambda_{2}\right)$. If $k \in F_{i}, l \in F_{j}, i \neq j$ and $F_{i}$ and $F_{j}$ have a connecting edge, then $\beta_{k}\left(\lambda_{2}\right) \neq \beta_{l}\left(\lambda_{2}\right)$ or $s_{k}\left(\lambda_{2}\right) \neq s_{l}\left(\lambda_{2}\right)$ for all penalty parameters in an interval $\left(\lambda_{2}, \lambda_{2}+\varepsilon\right)$ for some $\varepsilon>0$.

4. If $k, l \in F_{i}$ then $k$ and $l$ are connected in $\mathcal{G}$ by only going over nodes in $F_{i}$, i.e. $k, l$ are connected in $\left.\mathcal{G}\right|_{F_{i}}$, the subgraph of $\mathcal{G}$ induced by $F_{i}$.

This definition is almost the same as Definition 2 except for a small change in point 3 . This change reflects the adjustment for inactive coefficients as mentioned above. In addition to this, we also need a more formal definition of what active and inactive sets are.

Definition 4 For penalty parameter $\lambda_{2}^{0}$ (fixing $\lambda_{1}$ ), let $\mathcal{A}\left(\lambda_{2}^{0}\right)$ be the set of active fused sets and $\mathcal{N}\left(\lambda_{2}^{0}\right)$ be the set of inactive fused sets. Then

$$
\mathcal{A}\left(\lambda_{2}^{0}\right)=\left\{i \mid \beta_{F_{i}}(\lambda) \neq 0 \text { for } \lambda \in\left(\lambda_{2}^{0}, \lambda_{2}^{0}+\varepsilon\right)\right\}
$$

and

$$
\mathcal{N}\left(\lambda_{2}^{0}\right)=\left\{1, \ldots, p_{F}\left(\lambda_{2}^{0}\right)\right\} \backslash \mathcal{A}\left(\lambda_{2}^{0}\right)
$$

Here, a set is defined as active at $\lambda_{2}^{0}$ not if $\beta_{F_{i}}\left(\lambda_{2}^{0}\right) \neq 0$ but instead if $\beta_{F_{i}}\left(\lambda_{2}\right) \neq 0$ for $\lambda_{2}>\lambda_{2}^{0}$. There is only a difference between these two definitions at the breakpoints of the piecewise linear path. As we increase $\lambda$ from 0 to $\infty$, the definition for active sets was chosen to be "forward looking". This distinction is of a technical nature that is mostly important for the algorithm and proofs later.

Now that we have defined fused and active sets, we will determine how $\beta_{k}$ as well as $s_{k}$ and $t_{k l}$ change with $\lambda_{2}$.

\subsection{Derivation of the algorithm}

\subsubsection{The loss function}

The derivation of the algorithm will work similar to the sections before. First, we will incorporate the conditions of fused and active sets into the loss function and then find the derivative of $\beta_{F_{i}}\left(\lambda_{2}\right)$ with respect to $\lambda_{2}$. After this, we will determine how $s_{k}$ and $t_{k l}$ change with $\lambda_{2}$ and use it to specify when sets of variables are becoming active or inactive and have to be fused or split. Afterwards, we will prove that the solution is piecewise linear in $\lambda_{2}$ and assemble everything into a path algorithm.

Before going into more details, we need to define some additional notation. Using the sets of fused variables and active sets, we specify a predictor matrix that incorporates this information. Using the sets of fused variables $F_{i}$ for $i=1, \ldots, p_{F}\left(\lambda_{2}\right)$ define $\mathbf{X}^{F} \in \mathbb{R}^{n \times p_{F}\left(\lambda_{2}\right)}$ with

$$
\mathbf{x}_{i}^{F}=\sum_{k \in F_{i}} \mathbf{x}_{k}
$$

and based on this we incorporate the active sets into $\mathbf{X}^{F, \mathcal{A}} \in \mathbb{R}^{n \times\left|\mathcal{A}\left(\lambda_{2}\right)\right|}$ by dropping all columns in $\mathbf{X}^{F}$ that do not correspond to active sets. Similarly, by $\mathbf{X}^{\mathcal{A}}$ we refer to the columns of $\mathbf{X}$ corresponding to coefficients in active sets, i.e. $k \in F_{i}$ with $i \in \mathcal{A}\left(\lambda_{2}\right)$, and 
by $\mathbf{X}^{\mathcal{N}}$ to the columns of coefficients in inactive sets. Then the constrained loss function incorporating active and fused sets is

$$
\begin{aligned}
L_{F, \mathcal{A}, \lambda_{1}, \lambda_{2}}(\mathbf{y}, \mathbf{X}, \boldsymbol{\beta}) & =\frac{1}{2}\left(\mathbf{y}-\mathbf{X}^{F} \boldsymbol{\beta}^{F}\right)^{T}\left(\mathbf{y}-\mathbf{X}^{F} \boldsymbol{\beta}^{F}\right)+\lambda_{1} \sum_{i \in \mathcal{A}\left(\lambda_{2}\right)}\left|F_{i}\right|\left|\beta_{i}^{F}\right|+ \\
& +\lambda_{2} \sum_{i<j}\left|\left\{(k, l): k \in F_{i} l \in F_{j}\right\}\right|\left|\beta_{i}^{F}-\beta_{j}^{F}\right|
\end{aligned}
$$

where $\boldsymbol{\beta}^{F}$ is a vector such that $\beta_{i}^{F}=\beta_{F_{i}}$. Assuming that the fused and active sets are correct, the minimizer of $L_{F, \mathcal{A}, \lambda_{1}, \lambda_{2}}$ is also the minimizer of the original loss function $L_{\lambda_{1}, \lambda_{2}}$. Note that by definition of the fused sets $\beta_{F_{i}} \neq \beta_{F_{j}}$ (except for a finite number of breakpoints). Therefore, the subgradient equations for this constrained loss function with respect to $\lambda_{2}$ are

$$
\begin{aligned}
\frac{\partial L_{F, \mathcal{A}, \lambda_{1}, \lambda_{2}}(\mathbf{y}, \mathbf{X}, \boldsymbol{\beta})}{\partial \beta_{i}^{F}} & =-\mathbf{X}_{i}^{F, T} \mathbf{y}+\mathbf{X}_{i}^{F, T} \mathbf{X}^{F} \boldsymbol{\beta}^{F}+\lambda_{1}\left|F_{i}\right| s_{F_{i}}+ \\
& +\lambda_{2} \sum_{j \in \mathcal{A}\left(\lambda_{2}\right)}\left|\left\{(k, l): k \in F_{i} l \in F_{j}\right\}\right| \operatorname{sign}\left(\beta_{F_{i}}-\beta_{F_{j}}\right)=0,
\end{aligned}
$$

where $s_{F_{i}}$ is a short form for $s_{k}$ with $k \in F_{i}$ just as $\beta_{F_{i}}$. Now, if we define vectors $\mathbf{a}$ and $\mathbf{b}$ with

$$
a_{i}=\left|F_{i}\right| s_{F_{i}} \text { and } b_{i}=\sum_{j \neq i}\left|\left\{(k, l): k \in F_{i} l \in F_{j}\right\}\right| \operatorname{sign}\left(\beta_{F_{i}}-\beta_{F_{j}}\right)
$$

then we can write this optimality condition more compact as

$$
\frac{\partial L_{F, \mathcal{A}, \lambda_{2}}(\mathbf{y}, \mathbf{X}, \boldsymbol{\beta})}{\partial \boldsymbol{\beta}^{F}}=-\mathbf{X}^{F, T} \mathbf{y}+\mathbf{X}^{F, T} \mathbf{X}^{F} \boldsymbol{\beta}^{F}+\lambda_{1} \mathbf{a}+\lambda_{2} \mathbf{b}=0 .
$$

For $i \in \mathcal{A}\left(\lambda_{2}\right)$, we know that $s_{i}^{F}\left(\lambda_{2}\right)=s_{F_{i}}\left(\lambda_{2}\right)=1$ locally w.r.t. $\lambda_{2}$ and for $i \in \mathcal{N}\left(\lambda_{2}\right)$ we have $\beta_{i}^{F}\left(\lambda_{2}\right)=\beta_{F_{i}}\left(\lambda_{2}\right)=0$. Therefore, when taking derivatives with respect to $\lambda_{2}$ we get (splitting into active and inactive sets)

$$
\left(\mathbf{X}^{F, \mathcal{A}}\right)^{T} \mathbf{X}^{F, \mathcal{A}} \frac{\partial \boldsymbol{\beta}^{F, \mathcal{A}}}{\partial \lambda_{2}}+\mathbf{b}^{\mathcal{A}}=0
$$

as well as

$$
\left(\mathbf{X}^{F, \mathcal{N}}\right)^{T} \mathbf{X}^{F, \mathcal{A}} \frac{\partial \boldsymbol{\beta}^{F, \mathcal{A}}}{\partial \lambda_{2}}+\lambda_{1} \frac{\partial \mathbf{a}^{\mathcal{N}}}{\partial \lambda_{2}}+\mathbf{b}^{\mathcal{N}}=0
$$

We can solve this by

$$
\frac{\partial \boldsymbol{\beta}^{F, \mathcal{A}}}{\partial \lambda_{2}}=-\left(\left(\mathbf{X}^{F, \mathcal{A}}\right)^{T} \mathbf{X}^{F, \mathcal{A}}\right)^{-1} \mathbf{b}^{\mathcal{A}}
$$

and

$$
\frac{\partial \mathbf{a}^{\mathcal{N}}}{\partial \lambda_{2}}=-\frac{1}{\lambda_{1}}\left(\left(\mathbf{X}^{F, \mathcal{N}}\right)^{T} \mathbf{X}^{F, \mathcal{A}} \frac{\partial \boldsymbol{\beta}^{F, \mathcal{A}}}{\partial \lambda_{2}}+\mathbf{b}^{\mathcal{N}}\right)
$$

from which we get $\partial s_{F_{i}} / \partial \lambda_{2}=\left(1 /\left|F_{i}\right|\right) \cdot\left(\partial a_{i}^{\mathcal{N}} / \partial \lambda_{2}\right)$. Here, by the condition that $\operatorname{rank}(X)=p$, it is guaranteed that $\left(\left(\mathbf{X}^{F, \mathcal{A}}\right)^{T} \mathbf{X}^{F, \mathcal{A}}\right)^{-1}$ exists. Therefore, we have calculated the derivative of $\boldsymbol{\beta}^{F}$ and $\mathbf{s}^{F}$ from Equation (3). From this, we can derive the slope of the path for $\beta^{F}$ as well as 
determine when sets of coefficients become active or inactive. However, in order to see when to split a set, it remains to determine the behavior of $t_{k l}$ with respect to $\lambda_{2}$ or equivalently of $\tau_{k l}=\lambda_{2} t_{k l}$. We do this by inserting the solutions found above into the subgradient equations of the unconstrained problem (2). As before, we assume that we have a solution at $\lambda_{2}$ that satisfies the subgradient equations. Then for any coefficient $k \in F_{i}$ we have

$$
-\mathbf{x}_{k}^{T} \mathbf{y}+\mathbf{x}_{k}^{T} \mathbf{X}^{\mathcal{A}} \boldsymbol{\beta}^{\mathcal{A}}+\lambda_{1} s_{k}+\lambda_{2} \sum_{l:(k, l) \in E ; k, l \in F_{i}} t_{k l}+\lambda_{2} \sum_{l:(k, l) \in E ; l \notin F_{i}} t_{k l}=0
$$

again grouping the $t_{k l}$ by whether $k$ and $l$ belong to the same or different sets. When taking the derivative with respect to $\lambda_{2}$, we get

$$
\mathbf{x}_{k}^{T} \mathbf{X}^{\mathcal{A}} \frac{\partial \boldsymbol{\beta}^{\mathcal{A}}}{\partial \lambda_{2}}+\lambda_{1} \frac{\partial s_{k}}{\partial \lambda_{2}}+\sum_{l:(k, l) \in E ; k, l \in F_{i}} \frac{\partial \tau_{k l}}{\partial \lambda_{2}}+\sum_{l:(k, l) \in E ; l \notin F_{i}} t_{k l}=0
$$

In order to determine $\partial \tau_{k l} / \partial \lambda_{2}$, we use the maximum flow setup from the general FLSA algorithm as well as that $t_{k l}=\operatorname{sign}\left(\beta_{k}-\beta_{l}\right)$ for $k \in F_{i}, l \in F_{i}$. Then we define the push $p_{k}$ on node $k$ as

$$
p_{k}=-\mathbf{x}_{k}^{T} \mathbf{X}^{\mathcal{A}} \frac{\partial \boldsymbol{\beta}^{\mathcal{A}}}{\partial \lambda_{2}}-\lambda_{1} \frac{\partial s_{k}}{\partial \lambda_{2}}-\sum_{l:(k, l) \in E ; l \notin F_{i}} t_{k l}
$$

and therefore we have to solve

$$
p_{k}=\sum_{l:(k, l) \in E ; k, l \in F_{i}} \frac{\partial \tau_{k l}}{\partial \lambda_{2}} \text { for } k=1, \ldots, p
$$

This is exactly the same problem as in Section 3.2.2 and we use the same maximum flow setup described there to find $\partial \tau_{k l} / \partial \lambda_{2}$ by setting $\partial \tau_{k l} / \partial \lambda_{2}=f_{k l}$ where $f_{k l}$ is the maximal flow from node $k$ to node $l$ in $F_{i}$. Using all this, we can now show that the solution is piecewise linear in $\lambda_{2}$ in the following theorem:

Theorem 4 For some $\lambda_{2}^{0}$, let $F_{1}, \ldots, F_{p_{F}\left(\lambda_{2}^{0}\right)}$ be a valid grouping of the variables. Let $\tilde{\mathcal{G}}_{i}=\left(\tilde{V}_{i}, \tilde{E}_{i}, \tilde{C}_{i}\right)$ be the with $F_{i}$ associated maximum-flow graph as defined above. Also let $\beta_{k}\left(\lambda_{2}^{0}\right), s_{k}\left(\lambda_{2}^{0}\right)$ and $\tau_{k l}\left(\lambda_{2}^{0}\right)$ be a solution to the Fused Lasso problem for penalty parameter $\lambda_{2}=\lambda_{2}^{0}$. Assume that $\tilde{\mathcal{G}}_{i}$ has a maximum flow for which all flows coming from the source are at maximum capacity (i.e. $f_{r l}=c_{r l}$ for all $(r, l) \in \tilde{E}_{i}$ ), and $\partial \beta_{F_{i}} / \partial \lambda_{2}$ is as defined in Equation (5) as well as $\partial s_{k} / \partial \lambda_{2}$ as in (6). Then there exists some $\Delta>0$ such that for any $\lambda_{2} \in\left[\lambda_{2}^{0}, \lambda_{2}^{0}+\Delta\right]$, the solution to the Fused Lasso problem is given by

$$
\begin{aligned}
& \beta_{k}\left(\lambda_{2}\right)=\beta_{k}\left(\lambda_{2}^{0}\right)+\frac{\partial \beta_{F_{i}}}{\partial \lambda_{2}}\left(\lambda_{2}^{0}\right) \cdot\left(\lambda_{2}-\lambda_{2}^{0}\right) \quad \text { for } \quad k \in F_{i} \\
& s_{k}\left(\lambda_{2}\right)=s_{k}\left(\lambda_{2}^{0}\right)+\frac{\partial s_{F_{i}}}{\partial \lambda_{2}}\left(\lambda_{2}^{0}\right) \cdot\left(\lambda_{2}-\lambda_{2}^{0}\right) \quad \text { for } \quad k \in F_{i}
\end{aligned}
$$

and

$$
\tau_{k l}\left(\lambda_{2}\right)= \begin{cases}\tau_{k l}\left(\lambda_{2}^{0}\right)+f_{k l}\left(\lambda_{2}-\lambda_{2}^{0}\right) & \text { for } k, l \in F_{i} \\ \operatorname{sign}\left(\tau_{k l}\left(\lambda_{2}^{0}\right)\right) \lambda_{2} & \text { otherwise }\end{cases}
$$


The proof is very similar to the one for Theorem 3 and again can be found in Section 4.4. As before, we still have to define what $\Delta$ is.

\subsubsection{Activation and deactivation time}

In order to define $\Delta$, we have to find the value of $\lambda_{2}$ at which the fused sets themselves or their activation status changes. The hitting time $h\left(\lambda_{2}\right)$ and violation time $v\left(\lambda_{2}\right)$ are the same as in Section 3.2.4. In addition to this we also have to define the value of $\lambda_{2}$ at which sets are activated or deactivated. The activation time $\operatorname{act}\left(\lambda_{2}\right)$ is

$$
\operatorname{act}\left(\lambda_{2}\right)=\min _{i \in \mathcal{N}\left(\lambda_{2}\right)} \operatorname{act}_{i}\left(\lambda_{2}\right)
$$

where

$$
\operatorname{act}_{i}\left(\lambda_{2}\right)= \begin{cases}\frac{1-s_{F_{i}}}{\frac{\partial s_{F_{i}}}{\partial \lambda_{2}}}+\lambda_{2} & \text { if } \frac{\partial s_{F_{i}}}{\partial \lambda_{2}}>0 \\ \frac{s_{i}+1}{\partial s_{F_{i}}}+\lambda_{2} & \text { if } \frac{\partial s_{F_{i}}}{\partial \lambda_{2}}<0 \\ -\frac{\partial \lambda_{2}}{\infty} & \text { otherwise }\end{cases}
$$

is the activation time of set $F_{i}$ with $i \in \mathcal{N}\left(\lambda_{2}\right)$. The deactivation time on the other hand $d\left(\lambda_{2}\right)$ is

$$
d\left(\lambda_{2}\right)=\min _{i \in \mathcal{A}\left(\lambda_{2}\right)} d_{i}\left(\lambda_{2}\right)
$$

where

$$
d_{i}\left(\lambda_{2}\right)= \begin{cases}\frac{\beta_{F_{i}}}{-\frac{\partial \beta_{F_{i}}}{\partial \lambda_{2}}}+\lambda_{2} & \text { if } \frac{\partial \beta_{F_{i}}}{\partial \lambda_{2}}<0, \beta_{F_{i}}>0 \\ \frac{-\beta_{F_{i}}}{\partial \beta_{F_{i}}}+\lambda_{2} & \text { if } \frac{\partial \beta_{F_{i}}}{\partial \lambda_{2}}>0, \beta_{F_{i}}<0 \\ \infty & \text { otherwise }\end{cases}
$$

is the deactivation time of the active set $F_{i}$. Putting all this together, the length of the linear segment to the next breakpoint is

$$
\Delta\left(\lambda_{2}\right)=\min \left\{h\left(\lambda_{2}\right), v\left(\lambda_{2}\right), \operatorname{act}\left(\lambda_{2}\right), d\left(\lambda_{2}\right)\right\}-\lambda_{2} .
$$

Therefore, we have now defined all the necessary information on the previous theorem.

\subsubsection{Changing sets of variables}

What remains to be done is to find the rule on how to split and fuse sets of variables as well as how to activate or inactivate a set. First, the rule for splitting and fusing sets is very similar to the one in Section 3.2.4 with the only change being in step one to take the $s_{k}$ into account so that inactive sets can also be fused.

1. If there are sets $F_{i}$ and $F_{j}$ for which $\exists k \in F_{i}$ and $l \in F_{j}$ with $(k, l) \in E$ and $\beta_{F_{i}}=\beta_{F_{j}}$ and $s_{F_{i}}=s_{F_{j}}$, then fuse these sets into a new set $\tilde{F}_{i j}=F_{i} \cup F_{j}$ if $\frac{\partial \beta_{F_{i}}}{\partial \lambda_{2}}-\frac{\partial \beta_{F_{j}}}{\partial \lambda_{2}} \leq 0$, $\frac{\partial s_{F_{i}}}{\partial \lambda_{2}}-\frac{\partial s_{F_{j}}}{\partial \lambda_{2}} \leq 0$ and $t_{k l}=1$.

2. If there is a set $F_{i}$ for which in the associated maximal flow graph not all edges coming from the source are at maximal capacity, then split $F_{i}$ in the two subsets $R_{i}$ and $S_{i}$ as 
described above.

3. Iterate steps 2 and 3 until nothing changes.

Second, the rule for activating and inactivating the sets is then:

1. If for an inactive set $F_{i}$ we have $\frac{\partial s_{F_{i}}}{\partial \lambda_{2}}>0$ if $s_{F_{i}}=1$ or $\frac{\partial s_{F_{i}}}{\partial \lambda_{2}}<0$ if $s_{F_{i}}=-1$, then activate set $F_{i}$.

2. If for an active set $F_{i}$ we have $\beta_{F_{i}}=0$ and $s_{F_{i}}=1, \frac{\partial \beta_{F_{i}}}{\partial \lambda_{2}}<0$ or $s_{F_{i}}=-1, \frac{\partial \beta_{F_{i}}}{\partial \lambda_{2}}>0$, then deactivate the set.

When we use these two rules for values of $\lambda_{2}$ at which we have to adapt the fused and active sets, then the resulting sets will be valid. More precisely, the following proposition will hold:

Proposition 2 Assume that sets of variables $F_{i}, i=1, \ldots, p_{F}\left(\lambda_{2}-\right.$ ) are given (where $\lambda_{2}-$ denotes a limit from the left) with optimal values of $\beta_{F_{i}}\left(\lambda_{2}\right), s_{F_{i}}\left(\lambda_{2}\right)$ and $t_{k l}\left(\lambda_{2}\right)$ for all $k, l$. Furthermore assume that $\partial \beta_{F_{i}} / \partial \lambda_{2}$ and $\partial s_{F_{i}} / \partial \lambda_{2}$ are calculated as defined in equations (5) and (6) using the sets $F_{i}$. When using the two rules above for changing the fused sets and the activity status, the resulting sets will be valid according to Definition 4.1 and 4.1 .

The proof can be found in Section 4.5. This proposition completes the necessary steps for the path algorithm and we can now present an outline of the whole algorithm.

\subsection{Outline of the algorithm}

The algorithm is an extension of the FLSA algorithm that incorporates the active and inactive sets. In the case of the general FLSA algorithm, finding the starting value for $\lambda_{2}=0$ was particularly easy. For general Fused Lasso, finding the starting value needs an additional step. For $\lambda_{2}=0$, the loss function is

$$
L_{\lambda_{1}, 0}(\mathbf{y}, \mathbf{X}, \boldsymbol{\beta})=\frac{1}{2}(\mathbf{y}-\mathbf{X} \boldsymbol{\beta})^{T}(\mathbf{y}-\mathbf{X} \boldsymbol{\beta})+\lambda_{1} \sum_{k=1}^{p}\left|\beta_{k}\right|
$$

which is just the loss function of the regular Lasso. Therefore, we can find the starting values for $\beta$ by solving a regular Lasso problem. In order to find the starting value for $s$, we have to look at the subgradient equations:

$$
\frac{\partial L_{\lambda_{1}, \lambda_{2}}}{\partial \beta_{k}}=-\left(\mathbf{X}^{T} \mathbf{y}\right)_{k}+\left(\mathbf{X}^{T} \mathbf{X} \boldsymbol{\beta}\right)_{k}+\lambda_{1} s_{k}=0
$$

and thus

$$
s=\frac{1}{\lambda_{1}}\left(\mathbf{X}^{T} \mathbf{y}-\mathbf{X}^{T} \mathbf{X} \boldsymbol{\beta}\right) .
$$

Using these starting values we now have the complete algorithm which can be seen in Algorithm 1.

Most of the components of this algorithm are very similar to the FLSA algorithm. However, many efficiency gains that we could get in the FLSA case are not possible for the general Fused Lasso. For the FLSA, the derivative of $\beta_{F_{i}}$ and $t_{k l}$ for $k, l \in F_{i}$ only had to be updated 


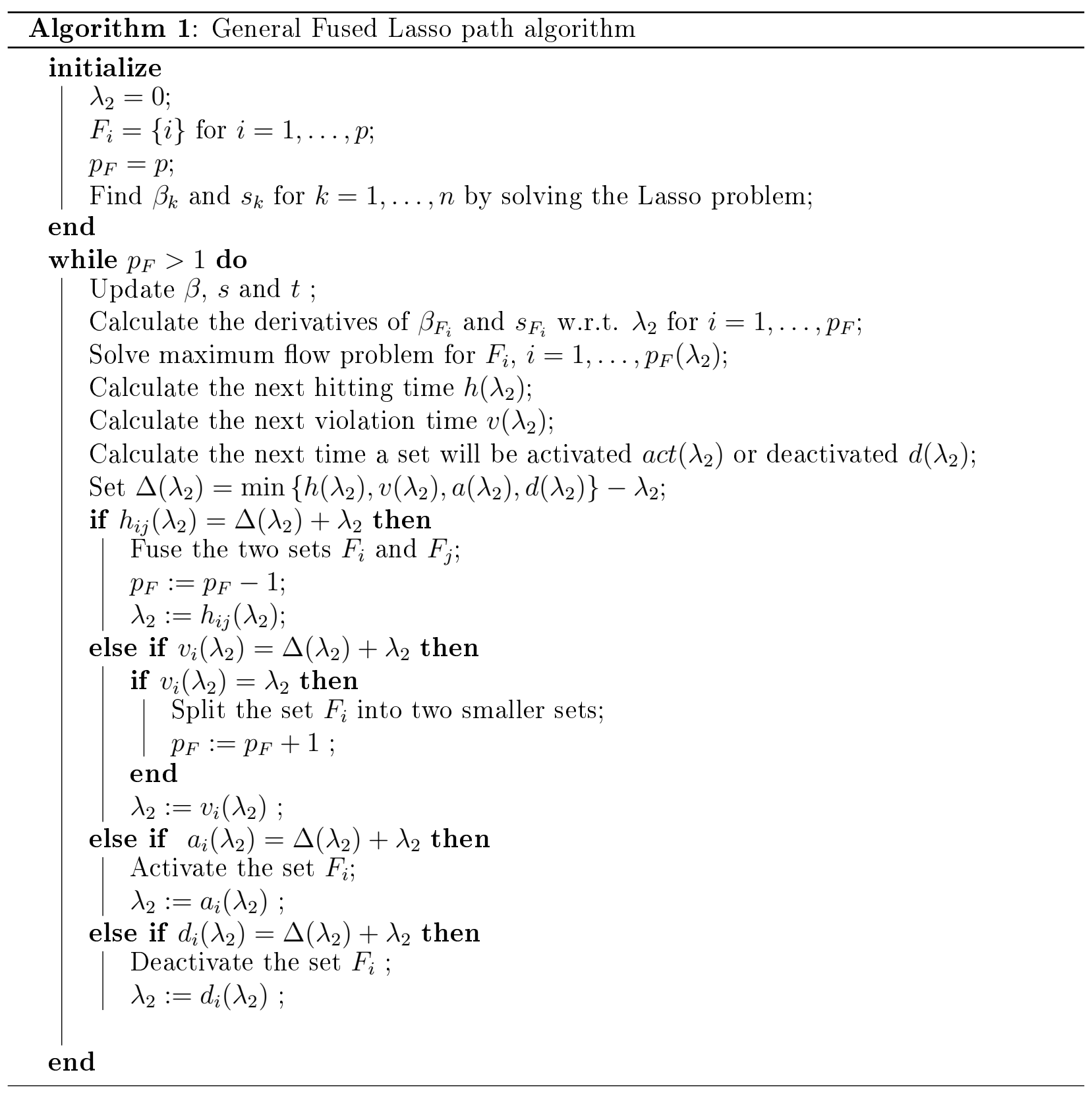


if $F_{i}$ was the result of the last split or fusion. For the Fused Lasso on the other hand, if only one of the active groups changes its composition, the path for all other groups are affected as well and have to be recomputed, including the maximum flow problems. Only inactive groups can be treated as in the FLSA case. Another computational bottleneck is the calculation of $\left(\left(\mathbf{X}^{F, \mathcal{A}}\right)^{T} \mathbf{X}^{F, \mathcal{A}}\right)^{-1}$. It is worthwhile to note that it is not necessary to recompute the whole matrix in every step. As from one step to the next, only very few columns of $\mathbf{X}^{F, \mathcal{A}}$ change, it is possible to get the new matrix inverse by up- and downdating the old matrix inverse.

Overall, the General Fused Lasso algorithm is computationally a lot more complex than the FLSA case. Apart from this, the more immediate applications are for the FLSA, especially the one-dimensional case. Therefore, we will only publish implementations for the FLSA cases at the moment and delay a program that solves the general version of the Fused Lasso to a later date.

\subsection{Proof of Theorem 4.2.1}

Proof. By the assumption, the values for $\beta_{F_{i}}\left(\lambda_{2}^{0}\right), s_{F_{i}}\left(\lambda_{2}^{0}\right)$ and $t_{k l}\left(\lambda_{2}^{0}\right)$ satisfy the subgradient equations (2) for the value $\lambda_{2}=\lambda_{2}^{0}$. Then the subgradient equations for value $\lambda_{2} \in\left[\lambda_{2}^{0}, \lambda_{2}^{0}+\Delta\right]$ are

$$
\begin{aligned}
& -\left(\mathbf{X}^{T} \mathbf{y}\right)_{k}+\left(\mathbf{X}^{T} \mathbf{X} \boldsymbol{\beta}\left(\lambda_{2}\right)\right)_{k}+\lambda_{1} s_{k}\left(\lambda_{2}\right)+\lambda_{2} \sum_{l:(k, l) \in E} t_{k l}\left(\lambda_{2}\right) \\
& =-\mathbf{x}_{k}^{T} \mathbf{y}+\mathbf{x}_{k}^{T} \mathbf{X} \boldsymbol{\beta}\left(\lambda_{2}^{0}\right)+\lambda_{1} s_{k}\left(\lambda_{2}^{0}\right)+\sum_{l:(k, l) \in E, l \notin F_{i}} \tau_{k l}\left(\lambda_{2}^{0}\right)+\sum_{l:(k, l) \in E, l \in F_{i}} \tau_{k l}\left(\lambda_{2}^{0}\right) \\
& +\left(\lambda_{2}-\lambda_{2}^{0}\right)\left(\mathbf{x}_{k}^{T} \mathbf{X} \frac{\partial \boldsymbol{\beta}}{\partial \lambda_{2}}+\lambda_{1} \frac{\partial s_{k}}{\partial \lambda_{2}}+\sum_{l:(k, l) \in E, l \notin F_{i}} t_{k l}\left(\lambda_{2}^{0}\right)+\sum_{l:(k, l) \in E, l \in F_{i}} f_{k l}\right) \\
& =0
\end{aligned}
$$

and we have to show that they are equal to zero. As by assumption $\boldsymbol{\beta}\left(\lambda_{2}^{0}\right), \mathbf{s}\left(\lambda_{2}^{0}\right)$ and $\boldsymbol{\tau}\left(\lambda_{2}^{0}\right)$ are by assumption optimal, it suffices to show that for all $k$

$$
\mathbf{x}_{k}^{T} \mathbf{X} \frac{\partial \boldsymbol{\beta}}{\partial \lambda_{2}}+\lambda_{1} \frac{\partial s_{k}}{\partial \lambda_{2}}+\sum_{l:(k, l) \in E, l \notin F_{i}} t_{k l}\left(\lambda_{2}^{0}\right)+\sum_{l:(k, l) \in E, l \in F_{i}} f_{k l}=0 .
$$

By the definition of the push on node $k$, this in turn is equivalent to

$$
-p_{k}+\sum_{l:(k, l) \in E, l \in F_{i}} f_{k l}=0
$$

In order to see this, observe that by assumption in the theorem, all nodes coming from the source are at maximum capacity. Furthermore, note that as $t_{k l}=-t_{l k}$, we also have that

$$
\begin{aligned}
\sum_{k \in F_{i}} p_{k} & =-\left(\mathbf{X}_{i}^{F}\right)^{T} \mathbf{X}^{\mathcal{A}} \frac{\partial \boldsymbol{\beta}^{\mathcal{A}}}{\partial \lambda_{2}}-\lambda_{1}\left|F_{i}\right| \frac{\partial s_{F_{i}}}{\partial \lambda_{2}} \\
& -\sum_{j \neq i}\left|\left\{(k, l): k \in F_{i}, l \in F_{j}\right\}\right| \operatorname{sign}\left(\beta_{F_{i}}-\beta_{F_{j}}\right)=0
\end{aligned}
$$


as can be seen in the derivation of the algorithm. Therefore,

$$
\sum_{k \in F_{i}, p_{k}>0} p_{k}=-\sum_{k \in F_{i}, p_{k}<0} p_{k}
$$

and thus, if all flows coming from the source are at capacity, so are all flows going into the sink. As the flows going into and out of every regular node $k$ in the maximum flow problem sum up to 0 , this just implies that for every node $k$

$$
-p_{k}+\sum_{l:(k, l) \in E, l \in F_{i}} f_{k l}=0
$$

holds. And this finishes the proof of the theorem.

\subsection{Proof of Proposition 4.2.3}

Proof. This proof is very similar to the proof of Proposition 2 above.

Before we start with the main part, note first that $\boldsymbol{\beta}\left(\lambda_{2}\right)$ is a continuous function of $\lambda_{2}$. Under the condition that $\operatorname{rank}(X)=p$, the loss function $L_{\lambda_{1}, \lambda_{2}}(\mathbf{y}, \mathbf{X}, \boldsymbol{\beta})$ is strictly convex in $\boldsymbol{\beta}$ and affine in $\lambda_{2}$. From this we can immediately see that $\min _{\boldsymbol{\beta}} L_{\lambda_{1}, \lambda_{2}}(\mathbf{y}, \mathbf{X}, \boldsymbol{\beta})$ is a continuous function of $\lambda_{2}$. However, then the strict convexity in $\boldsymbol{\beta}$ of $L$ implies the continuity of the solution $\boldsymbol{\beta}\left(\lambda_{2}\right)$.

Now back to the actual proof. In order for a set $F_{i}$ to be valid at $\lambda_{2}^{0}$, it has to hold that for any other set $F_{j}$ that $\beta_{F_{i}}\left(\lambda_{2}\right) \neq \beta_{F_{j}}\left(\lambda_{2}\right)$ or $s_{F_{i}}\left(\lambda_{2}\right) \neq s_{F_{j}}\left(\lambda_{2}\right)$ for $\lambda_{2} \in\left(\lambda_{2}^{0}, \lambda_{2}^{0}+\varepsilon\right)$ for some $\varepsilon>0$.

If $\beta_{F_{i}}\left(\lambda_{2}^{0}\right) \neq \beta_{F_{j}}\left(\lambda_{2}^{0}\right)$ or $s_{F_{i}}\left(\lambda_{2}^{0}\right) \neq s_{F_{j}}\left(\lambda_{2}^{0}\right)$, then this clearly holds as $\beta$ and $s$ are continuous in $\lambda_{2}$.

If $\beta_{F_{i}}=\beta_{F_{j}}$ and $s_{F_{i}}=s_{F_{j}}$, then the two sets will be fused unless one of the conditions $\partial \beta_{F_{i}} / \partial \lambda_{2}-\partial \beta_{F_{j}} / \partial \lambda_{2} \leq 0, \partial s_{F_{i}} / \partial \lambda_{2}-\partial s_{F_{j}} / \partial \lambda_{2} \leq 0$ or $t_{k l}=1$ is violated. First, as $F_{i}$ and $F_{j}$ are different sets, we have that $t_{k l}= \pm 1$ and as $t_{k l}=-t_{l k}$ by definition, we can assume without loss of generality that $t_{k l}=1$. If either one of the other two conditions fails, then either $\beta_{F_{i}}\left(\lambda_{2}\right)>\beta_{F_{j}}\left(\lambda_{2}\right)$ or $s_{F_{i}}\left(\lambda_{2}\right)>s_{F_{j}}\left(\lambda_{2}\right)$ for $\lambda_{2} \in\left(\lambda_{2}^{0}, \lambda_{2}^{0}+\varepsilon\right)$ and it is also not violating the restriction that $t_{k l}=\operatorname{sign}\left(\beta_{k}-\beta_{l}\right)$ for $\beta_{k} \neq \beta_{l}$ where $(k, l) \in E$ with $k \in F_{i}$ and $l \in F_{j}$. Therefore, the set is valid.

Another concern that we have to address is that we have to ensure the existence of $t_{k l}$ that satisfy the constraints $t_{k l} \in[-1,1]$ for $k, l \in F_{i}$ for all $i$. However, if a flow exists in $\tilde{\mathcal{G}}_{i}$, by the construction of the flow graph, this holds.

Therefore, for the fusing and splitting steps, it remains to show that there cannot be an infinite loop of fusions and splits. As there is only a finite number of nodes, it is enough to show that if two sets $F_{i}$ and $F_{j}$ have just been merged, they cannot be split again into $F_{i}$ and $F_{j}$ immediately at $\lambda_{2}=\lambda_{2}^{0}$.

In order to do this, assume that there is only one breakpoint exactly at $\lambda_{2}=\lambda_{2}^{0}$. Otherwise let $\mathbf{e} \sim N\left(0, I_{n}\right)$ and perturb $\mathbf{y}$ by using $\mathbf{y}+c \mathbf{e}$ where $c>0$ and send $c \rightarrow 0$. The probability of two breakpoints occurring for the same $\lambda_{2}$ for the random $\mathbf{y}+c \mathbf{e}$ is 0 and the solutions are all linear in $c$, thus the solution if just $\mathbf{y}$ is being used is equal to the limit of the solution using $\mathbf{y}+c \mathbf{e}$ for $c \rightarrow 0$.

Therefore, we can without loss of generality assume that there is only one breakpoint. Also, we assume that $\beta_{F_{i}}\left(\lambda_{2}^{0}\right)=\beta_{F_{j}}\left(\lambda_{2}^{0}\right)$ as well as $\beta_{F_{i}}\left(\lambda_{2}\right)>\beta_{F_{j}}\left(\lambda_{2}\right)$ for $\lambda_{2} \in\left(\lambda_{2}^{0}-\varepsilon, \lambda_{2}^{0}\right)$ and 
therefore $\partial \beta_{F_{i}} / \partial \lambda_{2}<\partial \beta_{F_{j}} / \partial \lambda_{2}$ (the same remains true when we use s instead of $\boldsymbol{\beta}$ ). This implies that the two groups $F_{i}$ and $F_{j}$ have to remain fused for at least an interval $\left[\lambda_{2}^{0}, \lambda_{2}^{1}\right]$ for $\lambda_{2}^{0}<\lambda_{2}^{1}$. To see this, observe that there are only two other possible cases:

$\beta_{F_{i}}\left(\lambda_{2}\right)>\beta_{F_{j}}\left(\lambda_{2}\right)$ for $\lambda_{2}>\lambda_{2}^{0}$ : This would require that $\partial \beta_{F_{i}} / \partial \lambda_{2}\left(\lambda_{2}\right)>\partial \beta_{F_{j}} / \partial \lambda_{2}\left(\lambda_{2}\right)$ as $\beta_{F_{i}}\left(\lambda_{2}^{0}\right)=\beta_{F_{j}}\left(\lambda_{2}^{0}\right)$. However, this is not possible as in this case, equation (5) did not change compared to $\lambda_{2}<\lambda_{2}^{0}$. Therefore $\partial \beta_{F_{i}} / \partial \lambda_{2}<\partial \beta_{F_{j}} / \partial \lambda_{2}$ as before, contradicting $\beta_{F_{i}}\left(\lambda_{2}\right)>\beta_{F_{j}}\left(\lambda_{2}\right)$ for $\lambda_{2}>\lambda_{2}^{0}$.

$\beta_{F_{i}}\left(\lambda_{2}\right)<\beta_{F_{j}}\left(\lambda_{2}\right)$ for $\lambda_{2}>\lambda_{2}^{0}$ : In this case we would have that due to the continuity of $\beta$ it holds

$$
\lim _{\lambda_{2} \uparrow \lambda_{2}^{0}} \frac{\partial L_{F, \mathcal{A}, \lambda_{1}, \lambda_{2}}(\mathbf{y}, \mathbf{X}, \boldsymbol{\beta})}{\partial \beta_{i}^{F}}=\lim _{\lambda_{2} \downarrow \lambda_{2}^{0}} \frac{\partial L_{F, \mathcal{A}, \lambda_{1}, \lambda_{2}}(\mathbf{y}, \mathbf{X}, \boldsymbol{\beta})}{\partial \beta_{i}^{F}}+2 \lambda_{2}\left|\left\{(k, l): k \in F_{i, l} l \in F_{j}\right\}\right| .
$$

By assumption we know that $\boldsymbol{\beta}\left(\lambda_{2}\right)$ is optimal for $\lambda_{2}<\lambda_{2}^{0}$ and thus

$$
L_{F, \mathcal{A}, \lambda_{1}, \lambda_{2}}(\mathbf{y}, \mathbf{X}, \boldsymbol{\beta})=0 \quad \text { for } \quad \lambda_{2}<\lambda_{2}^{0} .
$$

However this implies then that $\boldsymbol{\beta}\left(\lambda_{2}\right)$ for which $\beta_{F_{i}}\left(\lambda_{2}\right)<\beta_{F_{j}}\left(\lambda_{2}\right)$ can not be optimal for $\lambda_{2}>\lambda_{2}^{0}$, again contradicting the assumption.

This shows that a fused group $F_{i} \cup F_{j}$ has to remain fused for an interval $\left[\lambda_{2}^{0}, \lambda_{2}^{1}\right]$. However, for our algorithm it is necessary that then a flow exists for which all flows coming from the source are at maximum capacity. As the grouping with $F_{i} \cup F_{j}$ is optimal for $\left[\lambda_{2}^{0}, \lambda_{2}^{1}\right]$, using the subgradient equations (7) as well as the linearity of $\boldsymbol{\beta}^{\mathcal{A}}$ and $\mathbf{s}$ in $\lambda_{2}$ and that $t_{k l}=$ const. for $(k, l) \in E, k \in F_{i} \cup F_{j}$ and $l \notin F_{i} \cup F_{j}$ we have that the $\tau_{k l}$ for $(k, l) \in E ; k, l \in F_{i} \cup F_{j}$ have to satisfy a linear equation of the form

$$
\sum_{(k, l) \in E ; l \in F_{i} \cup F_{j}} \tau_{k l}+\lambda_{2} c_{k}=d_{k} \quad \text { for } \quad k \in F_{i} \cup F_{j}
$$

for some vectors $c_{k}$ and $d_{k}$. As the grouping is optimal, we also know that a solution exists for every $\lambda_{2} \in\left[\lambda_{2}^{0}, \lambda_{2}^{1}\right]$. The space of all solutions of this linear equation is a vector space and thus we know that there exist linear functions $\tau_{k l}\left(\lambda_{2}\right)$ that satisfy these equations. But then, the flows $f_{k l}=\partial \tau_{k l} / \partial \lambda_{2}$ satisfy the maximum flow problem of the graph $\tilde{\mathcal{G}}_{F_{i} \cup F_{j}}$ with all flows from the source at maximum capacity (as the maximum flow problem solves the above equations after taking the derivative w.r.t. $\lambda_{2}$ ). Therefore, the group $F_{i} \cup F_{j}$ will not be forced to break up by our algorithm.

Now the only thing that remains to do is to make sure that the rules for activating and deactivating sets are correct. In order to see this, we again use the continuity and piecewise linearity of $\boldsymbol{\beta}$ w.r.t. $\lambda_{2}$. It is easy to see, that this also implies continuity and piecewise linearity for $\mathbf{s}$ w.r.t. $\lambda_{2}$.

Activate an inactive set: Let $F_{i}$ be the inactive where w.l.o.g. $s_{F_{i}}\left(\lambda_{2}^{0}\right)=1$ as well as $\beta_{F_{i}}\left(\lambda_{2}^{0}\right)=0$ and $\left(\partial s_{F_{i}} / \partial \lambda_{2}\right)\left(\lambda_{2}^{0}\right)>0$. First, the set cannot remain inactive for $\lambda_{2}>\lambda_{2}^{0}$, as then we would have $\left(\partial s_{F_{i}} / \partial \lambda_{2}\right)\left(\lambda_{2}^{0}\right) \leq 0$, which is a contradiction. Also $\left(\partial \beta_{F_{i}} / \partial \lambda_{2}\right)\left(\lambda_{2}\right)<$ 0 is not possible, as then $s\left(\lambda_{2}\right)=-1$ for $\lambda_{2}>\lambda_{2}^{0}$, which would violate the continuity of s. Therefore, $\left(\partial \beta_{F_{i}} / \partial \lambda_{2}\right)\left(\lambda_{2}\right)>0$ and thus $\beta_{F_{i}}\left(\lambda_{2}\right)>0$ for $\lambda_{2}>\lambda_{2}^{0}$ and then $F_{i}$ satisfies the activity condition. 
Deactivate an active set: Let $F_{i}$ be active where w.l.o.g. $s_{F_{i}}\left(\lambda_{2}^{0}\right)=1, \beta_{F_{i}}\left(\lambda_{2}^{0}\right)=0$ and $\left(\partial \beta_{F_{i}} / \partial \lambda_{2}\right)\left(\lambda_{2}^{0}\right)<0$. Then due to the negative derivative, we have $\beta_{F_{i}}\left(\lambda_{2}\right) \leq 0$ for $\lambda_{2}>\lambda_{2}^{0}$. However, if $\beta_{F_{i}}\left(\lambda_{2}\right)<0$, then $s_{F_{i}}\left(\lambda_{2}\right)=-1$, again violating the continuity of s. Thus, we have that $\beta_{F_{i}}\left(\lambda_{2}\right)=0$ for $\lambda_{2} \in\left(\lambda_{2}^{0}, \lambda_{2}^{0}+\varepsilon\right)$ and therefore, the condition for being active is not satisfied for $F_{i}$. Thus it is inactive.

\section{References}

Bradley Efron, Trevor Hastie, Ian Johnstone, and Robert Tibshirani. Least angle regression. Annals of Statistics, 32(2):407-499, 2004.

J. Friedman, T. Hastie, H. Hoefling, and R. Tibshirani. Pathwise coordinate optimization. Annals of Applied Statistics, 2007. 